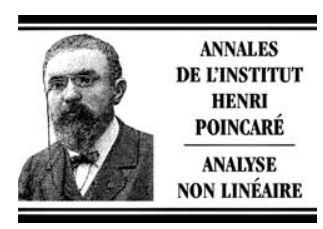

www.elsevier.com/locate/anihpc

\title{
A nonlinear model for inextensible rods as a low energy $\Gamma$-limit of three-dimensional nonlinear elasticity
}

\section{Un modèle non linéaire de poutre inextensionnelle comme $\Gamma$-limite de l'elasticité non linéaire tridimensionnelle}

\author{
Maria Giovanna Mora, Stefan Müller \\ Max-Planck Institute for Mathematics in the Sciences, Inselstr. 22-26, 04103 Leipzig, Germany \\ Received 17 February 2003 \\ Available online 24 October 2003
}

\begin{abstract}
Using a variational approach we rigorously deduce a nonlinear model for inextensible rods from three-dimensional nonlinear elasticity, passing to the limit as the diameter of the rod goes to zero. The theory obtained is analogous to the Föppl-von Kármán theory for plates. We also derive an asymptotic expansion of the solution and compare it to a similar expansion which Murat and Sili obtained starting from three-dimensional linear elasticity.

(C) 2004 L'Association Publications de l'Institut Henri Poincaré. Published by Elsevier B.V. All rights reserved
\end{abstract}

\section{Résumé}

Par une méthode variationnelle on dérive rigoureusement un modèle non linéaire de poutre inextensionelle. Le modèle est déduit de l'élasticité non linéaire tridimensionnelle, après une mise à l'échelle adéquate, en passant à la limite lorsque le diamètre de la poutre tend vers zéro. On obtient ainsi une théorie analogue à celle de Föppl-von Kármán pour les plaques. On dérive aussi une expansion asymptotique des solutions et on la compare avec une expansion similaire que Murat et Sili ont obtenue à partir de la théorie linéaire de l'élasticité.

(c) 2004 L'Association Publications de l'Institut Henri Poincaré. Published by Elsevier B.V. All rights reserved

MSC: 74K10; 49J45

\section{Introduction}

In this paper we continue the rigorous derivation of rod equations by three-dimensional nonlinear elasticity through $\Gamma$-convergence. We refer to [2,3] for a survey about one-dimensional models and a discussion on the history of the classical derivations of such theories (see also $[4,9,17]$ ).

E-mail addresses: mmora@mis.mpg.de (M.G. Mora), sm@mis.mpg.de (S. Müller).

0294-1449/\$ - see front matter (C) 2004 L'Association Publications de l'Institut Henri Poincaré. Published by Elsevier B.V. All rights reserved doi:10.1016/j.anihpc.2003.08.001 
Under the assumption of uniformly small strain Mielke rigorously derived the fully nonlinear rod equations through a centre manifold argument [10,11]. While Mielke's approach is based on a deep analysis of the equilibrium equations, the starting point of our approach is the elastic energy

$$
E^{(h)}(v):=\int_{\Omega_{h}} W\left(\left(z_{1}, \frac{z^{\prime}}{h}\right), \nabla v(z)\right) \mathrm{d} z
$$

of a deformation $v \in W^{1,2}\left(\Omega_{h} ; \mathbb{R}^{3}\right)$, where $\Omega_{h}:=(0, L) \times h S, S$ is an open subset of $\mathbb{R}^{2}$ with Lipschitz boundary, and $z:=\left(z_{1}, z^{\prime}\right)$ varies in $\Omega_{h}$. By heuristic arguments energies $E^{(h)}$ of order $h^{2}$ are expected to correspond to stretching and shearing deformations of the fibre, leading to a string theory, while energies $E^{(h)}$ of order $h^{4}$ to bending flexures and torsions keeping the fibre unextended, leading to a rod theory. If $E^{(h)}$ is of order $h^{6}$, one expects that the corresponding deformation is close to a rigid motion, so that one can linearize around it and obtain a theory analogous to the Föppl-von Kármán theory for plates (see [7]). The elastic theory for strings has been rigorously derived by Acerbi, Buttazzo and Percivale in [1], while the bending-torsion theory for inextensible rods has been recently justified in [12] and independently by Pantz in [16]. The mathematical setting in which both results are formulated is that of $\Gamma$-convergence (see [5] for a comprehensive introduction to this notion of variational convergence). In this paper we analyse the case where $E^{(h)}$ is of order $h^{6}$ and we identify the $\Gamma$-limit of the functionals $h^{-6} E^{(h)}$.

To state our results, it is convenient to introduce the following change of variables:

$$
z_{1}=x_{1}, \quad z^{\prime}=h x^{\prime},
$$

and to rescale deformations according to $y(x):=v(z(x))$, so that $y$ belongs to $W^{1,2}\left(\Omega ; \mathbb{R}^{3}\right)$, where $\Omega:=$ $(0, L) \times S$. We will use the notation

$$
\nabla_{h} y:=\left(y_{, 1}\left|\frac{1}{h} y_{, 2}\right| \frac{1}{h} y_{, 3}\right)
$$

So that

$$
\frac{1}{h^{2}} E^{(h)}(v)=I^{(h)}(y):=\int_{\Omega} W\left(x, \nabla_{h} y(x)\right) \mathrm{d} x .
$$

We assume that the stored energy function $W$ satisfies the following assumptions:

(i) $W: \Omega \times \mathbb{M}^{3 \times 3} \rightarrow[0,+\infty]$ is a Carathéodory function; for some $\delta>0$ the function $F \mapsto W(x, F)$ is of class $C^{2}$ for $\operatorname{dist}(F, \operatorname{SO}(3))<\delta$ and for a.e. $x \in \Omega$

(ii) the second derivative $\partial^{2} W / \partial F^{2}$ is a Carathéodory function on the set $\Omega \times\left\{F \in \mathbb{M}^{3 \times 3}: \operatorname{dist}(F, \operatorname{SO}(3))<\delta\right\}$ and there exists a constant $\gamma>0$ such that

$$
\left|\frac{\partial^{2} W}{\partial F^{2}}(x, F)[G, G]\right| \leqslant \gamma|G|^{2} \quad \text { for } \operatorname{dist}(F, \operatorname{SO}(3))<\delta \text { and } G \in \mathbb{M}_{\text {sym }}^{3 \times 3} ;
$$

(iii) $W$ is frame-indifferent, i.e., $W(x, F)=W(x, R F)$ for a.e. $x \in \Omega$ and every $F \in \mathbb{M}^{3 \times 3}, R \in \mathrm{SO}(3)$;

(iv) $W(x, F)=0$ if $F \in \mathrm{SO}(3) ; W(x, F) \geqslant C \operatorname{dist}^{2}(F, \mathrm{SO}(3))$ for every $F \in \mathbb{M}^{3 \times 3}$, where the constant $C>0$ is independent of $x$.

Under these assumptions we first show a compactness result for sequences of deformations whose rescaled energies $h^{-4} I^{(h)}$ are bounded. More precisely, we prove in Theorem 2.2 that for any sequence $\left(y^{(h)}\right)$ such that

$$
\limsup _{h \rightarrow 0} \frac{1}{h^{4}} I^{(h)}\left(y^{(h)}\right)<+\infty,
$$


we can find some constants $\bar{R}^{(h)} \in \mathrm{SO}(3), c^{(h)} \in \mathbb{R}$ such that $\bar{R}^{(h)} \rightarrow \bar{R}$ and the functions $\tilde{y}^{(h)}:=\left(\bar{R}^{(h)}\right)^{\mathrm{T}} y^{(h)}-$ $c^{(h)}$ satisfy (up to subsequences)

$$
\nabla_{h} \tilde{y}^{(h)} \rightarrow \text { Id } \quad \text { strongly in } L^{2}(\Omega) .
$$

Since the limit deformation is a rigid motion, it is natural to study the behaviour of the deviation (suitably rescaled) of $\tilde{y}^{(h)}$ from the identity. Thus, we introduce the functions

$$
\begin{aligned}
& u^{(h)}\left(x_{1}\right):=\int_{S} \frac{\tilde{y}_{1}^{(h)}(x)-x_{1}}{h^{2}} \mathrm{~d} x_{2} \mathrm{~d} x_{3}, \quad v_{k}^{(h)}\left(x_{1}\right):=\int_{S} \frac{\tilde{y}_{k}^{(h)}(x)}{h} \mathrm{~d} x_{2} \mathrm{~d} x_{3} \quad \text { for } k=2,3, \\
& w^{(h)}\left(x_{1}\right):=\frac{1}{\mu(S)} \int_{S} \frac{x_{2} \tilde{y}_{3}^{(h)}(x)-x_{3} \tilde{y}_{2}^{(h)}(x)}{h^{2}} \mathrm{~d} x_{2} \mathrm{~d} x_{3},
\end{aligned}
$$

where we have set $\mu(S):=\int_{S}\left(x_{2}^{2}+x_{3}^{2}\right) \mathrm{d} x_{2} \mathrm{~d} x_{3}$ and we have chosen the axes in such a way that

$$
\int_{S} x_{2} x_{3} \mathrm{~d} x_{2} \mathrm{~d} x_{3}=\int_{S} x_{2} \mathrm{~d} x_{2} \mathrm{~d} x_{3}=\int_{S} x_{3} \mathrm{~d} x_{2} \mathrm{~d} x_{3}=0 .
$$

The function $u^{(h)}$ measures the averaged deviation of the deformation component along the fibre, while $v_{k}^{(h)}$ the averaged deviation of the deformation components which are normal to the fibre. The function $w^{(h)}$ is related to the twist of the cross section. In Theorem 2.2 we show that (up to subsequences) the following properties hold:

- $u^{(h)} \rightarrow u$ weakly in $W^{1,2}(0, L)$;

- $v_{k}^{(h)} \rightarrow v_{k}$ strongly in $W^{1,2}(0, L)$, where $v_{k} \in W^{2,2}(0, L)$ for $k=2,3$;

- $w^{(h)} \rightarrow w$ weakly in $W^{1,2}(0, L)$.

In Theorem 4.5 we prove that the $\Gamma$-limit of the functionals $h^{-4} I^{(h)}$ is an integral functional depending on $u, v_{k}$, and $w$, of the following form:

$$
I^{0}\left(u, v_{2}, v_{3}, w\right)=\frac{1}{2} \int_{0}^{L} Q\left(x_{1}, u_{, 1}+\frac{1}{2}\left(v_{2,1}^{2}+v_{3,1}^{2}\right), A_{, 1}\right) \mathrm{d} x_{1},
$$

where

$$
A:=\left(\begin{array}{ccc}
0 & -v_{2,1} & -v_{3,1} \\
v_{2,1} & 0 & -w \\
v_{3,1} & w & 0
\end{array}\right),
$$

and $Q\left(x_{1}, t, F\right)$ is a quadratic form in the pair $(t, F)$ defined through a suitable minimization procedure involving the quadratic form of linearized elasticity $Q_{3}(x, G):=\frac{\partial^{2} W}{\partial F^{2}}(x, \mathrm{Id})[G, G]$ (see (4.1)).

A key ingredient in the proof is a rigidity result by Friesecke, James, and Müller (see Theorem 2.1), which ensures that low energy deformations are close to a rigid motion and provides the crucial estimate in the proof of compactness.

In the last part of the paper we also show (under slight additional regularity assumptions) that solutions $\tilde{y}^{(h)}$ admit an asymptotic development $\hat{y}^{(h)}$ of the form

$$
\begin{aligned}
& \hat{y}_{1}^{(h)}=x_{1}+h^{2}\left(u-x_{2} v_{2,1}-x_{3} v_{3,1}\right)+h^{3} \beta_{1}, \\
& \hat{y}_{k}^{(h)}=h x_{k}+h v_{k}+h^{2} w x_{k}^{\perp}+h^{3} \beta_{k} \quad \text { for } k=2,3,
\end{aligned}
$$


where $\beta \in L^{2}\left(\Omega ; \mathbb{R}^{3}\right)$ and $x^{\perp}$ denotes the point $\left(0,-x_{3}, x_{2}\right)$. The asymptotic expansion has to be interpreted in the following sense:

$$
\frac{\tilde{y}_{1}^{(h)}-\hat{y}_{1}^{(h)}}{h^{2}} \rightarrow 0, \quad \frac{\tilde{y}_{k}^{(h)}-\hat{y}_{k}^{(h)}}{h} \rightarrow 0 \quad(k=2,3) \text { in } W^{1,2}(\Omega),
$$

and

$$
\frac{\left[\left(\nabla_{h} \tilde{y}^{(h)}\right)^{\mathrm{T}} \nabla_{h} \tilde{y}^{(h)}\right]^{1 / 2}-\mathrm{Id}}{h^{2}}-\frac{\left[\left(\nabla_{h} \hat{y}^{(h)}\right)^{\mathrm{T}} \nabla_{h} \hat{y}^{(h)}\right]^{1 / 2}-\mathrm{Id}}{h^{2}} \rightarrow 0 \quad \text { in } L^{1}(\Omega) .
$$

This asymptotic analysis generalizes to the nonlinear setting an earlier result by Murat and Sili in the context of linearized elasticity (see $[13,14])$.

The plan of the paper is as follows. In Section 2 we prove the compactness result and a lower bound for the $\Gamma$-limit, while in Section 3 we show an upper bound; Section 4 contains the identification of the $\Gamma$-limit and some remarks about the characterization of the limit density $Q$ when $W$ satisfies some additional requirements, as homogeneity or isotropy; finally, Section 5 is devoted to the study of the asymptotic behaviour of solutions.

\section{Compactness and lower bound}

In the sequel $S$ is a bounded open subset of $\mathbb{R}^{2}$ with Lipschitz boundary. We assume that $\mathcal{L}^{2}(S)=1$. We recall that the axes are chosen in such a way that

$$
\int_{S} x_{2} x_{3} \mathrm{~d} x_{2} \mathrm{~d} x_{3}=\int_{S} x_{2} \mathrm{~d} x_{2} \mathrm{~d} x_{3}=\int_{S} x_{3} \mathrm{~d} x_{2} \mathrm{~d} x_{3}=0 .
$$

The following rigidity estimate is proved in [6].

Theorem 2.1. Let $U$ be a bounded Lipschitz domain in $\mathbb{R}^{n}, n \geqslant 2$. Then there exists a constant $C(U)$ with the following property: for every $v \in W^{1,2}\left(U ; \mathbb{R}^{n}\right)$ there is an associated rotation $R \in \operatorname{SO}(n)$ such that

$$
\|\nabla v-R\|_{L^{2}(U)} \leqslant C(U)\|\operatorname{dist}(\nabla v, \operatorname{SO}(n))\|_{L^{2}(U)} .
$$

Using the previous theorem we can show the following compactness result.

Theorem 2.2. Let $\left(y^{(h)}\right)$ be a sequence in $W^{1,2}\left(\Omega ; \mathbb{R}^{3}\right)$ such that

$$
\limsup _{h \rightarrow 0} \frac{1}{h^{4}} \int_{\Omega} W\left(x, \nabla_{h} y^{(h)}\right) \mathrm{d} x<+\infty .
$$

Then, there exist maps $R^{(h)}:[0, L] \rightarrow \mathrm{SO}(3), \widetilde{R}^{(h)}:[0, L] \rightarrow \mathbb{M}^{3 \times 3}$ with $\left|\widetilde{R}^{(h)}\right| \leqslant c$, and constants $\bar{R}^{(h)} \in$ $\mathrm{SO}(3), c^{(h)} \in \mathbb{R}$ such that the functions $\tilde{y}^{(h)}:=\left(\bar{R}^{(h)}\right)^{\mathrm{T}} y^{(h)}-c^{(h)}$ satisfy

$$
\begin{aligned}
& \left\|\nabla_{h} \tilde{y}^{(h)}-R^{(h)}\right\|_{L^{2}(\Omega)} \leqslant C h^{2}, \\
& \left\|R^{(h)}-\widetilde{R}^{(h)}\right\|_{L^{2}(0, L)} \leqslant C h^{2}, \quad\left\|\nabla \widetilde{R}^{(h)}\right\|_{L^{2}(0, L)} \leqslant C h, \\
& \left\|R^{(h)}-\operatorname{Id}\right\|_{L^{\infty}(0, L)} \leqslant C h .
\end{aligned}
$$

Moreover, if we define 


$$
\begin{aligned}
& u^{(h)}\left(x_{1}\right):=\int_{S} \frac{\tilde{y}_{1}^{(h)}(x)-x_{1}}{h^{2}} \mathrm{~d} x_{2} \mathrm{~d} x_{3}, \\
& v_{k}^{(h)}\left(x_{1}\right):=\int_{S} \frac{\tilde{y}_{k}^{(h)}(x)}{h} \mathrm{~d} x_{2} \mathrm{~d} x_{3} \quad \text { for } k=2,3, \\
& w^{(h)}\left(x_{1}\right):=\frac{1}{\mu(S)} \int_{S} \frac{x_{2} \tilde{y}_{3}^{(h)}(x)-x_{3} \tilde{y}_{2}^{(h)}(x)}{h^{2}} \mathrm{~d} x_{2} \mathrm{~d} x_{3},
\end{aligned}
$$

where $\mu(S):=\int_{S}\left(x_{2}^{2}+x_{3}^{2}\right) \mathrm{d} x_{2} \mathrm{~d} x_{3}$, then, up to subsequences, the following properties are satisfied:

(a) $u^{(h)} \rightarrow u$ weakly in $W^{1,2}(0, L)$;

(b) $v_{k}^{(h)} \rightarrow v_{k}$ strongly in $W^{1,2}(0, L)$, where $v_{k} \in W^{2,2}(0, L)$ for $k=2,3$;

(c) $w^{(h)} \rightarrow w$ weakly in $W^{1,2}(0, L)$;

(d) $\left(\nabla_{h} \tilde{y}^{(h)}-\mathrm{Id}\right) / h \rightarrow A$ strongly in $L^{2}(\Omega)$, where $A \in W^{1,2}\left((0, L) ; \mathbb{M}^{3 \times 3}\right)$ is given by

$$
A=\left(\begin{array}{ccc}
0 & -v_{2,1} & -v_{3,1} \\
v_{2,1} & 0 & -w \\
v_{3,1} & w & 0
\end{array}\right)
$$

(e) $\operatorname{sym}\left(R^{(h)}-\mathrm{Id}\right) / h^{2} \rightarrow A^{2} / 2$ uniformly on $(0, L)$;

(f) the sequence $\left(\beta^{(h)}\right)$ defined by

$$
\begin{aligned}
& \beta_{1}^{(h)}(x):=\frac{1}{h}\left(\frac{\tilde{y}_{1}^{(h)}(x)-x_{1}}{h^{2}}-u^{(h)}\left(x_{1}\right)+x_{2} v_{2,1}^{(h)}\left(x_{1}\right)+x_{3} v_{3,1}^{(h)}\left(x_{1}\right)\right), \\
& \beta_{j}^{(h)}(x):=\frac{1}{h^{2}}\left(\frac{\tilde{y}_{j}^{(h)}(x)-h x_{j}}{h}-v_{j}^{(h)}\left(x_{1}\right)-h w^{(h)}\left(x_{1}\right) x_{j}^{\perp}\right) \quad \text { for } j=2,3,
\end{aligned}
$$

where $x^{\perp}:=\left(0,-x_{3}, x_{2}\right)$, is weakly convergent in $L^{2}(\Omega)$ to a function $\beta$ belonging to the space

$$
\mathcal{B}:=\left\{\theta \in L^{2}\left(\Omega ; \mathbb{R}^{3}\right): \int_{S} \theta(x) \mathrm{d} x_{2} \mathrm{~d} x_{3}=0, \theta_{, 2}, \theta, 3 \in L^{2}\left(\Omega ; \mathbb{R}^{3}\right), \int_{S}\left(x_{3} \theta_{2}-x_{2} \theta_{3}\right) \mathrm{d} x_{2} \mathrm{~d} x_{3}=0\right\} .
$$

Moreover, $\beta_{, k}^{(h)} \rightarrow \beta_{, k}$ in $L^{2}(\Omega)$ for $k=2,3$.

Proof. The coerciveness assumption on $W$ and the bound (2.2) imply that

$$
\limsup _{h \rightarrow 0} \frac{1}{h^{4}} \int_{\Omega} \operatorname{dist}^{2}\left(\nabla_{h} y^{(h)}, \operatorname{SO}(3)\right) \mathrm{d} x<+\infty .
$$

Applying Theorem 2.1 as in the proof of the compactness result of [12], we can find a sequence of piecewise constant maps $R^{(h)}:[0, L] \rightarrow \mathrm{SO}(3)$ such that

$$
\int_{\Omega}\left|\nabla_{h} y^{(h)}-R^{(h)}\right|^{2} \mathrm{~d} x \leqslant C h^{4},
$$

and

$$
\int_{I^{\prime}}\left|R^{(h)}\left(x_{1}+\xi\right)-R^{(h)}\left(x_{1}\right)\right|^{2} \mathrm{~d} x_{1} \leqslant C h^{2}(|\xi|+h)^{2},
$$


where $I^{\prime}$ is any open interval compactly contained in $(0, L)$ and $\xi \in \mathbb{R}$ satisfies $|\xi| \leqslant \operatorname{dist}\left(I^{\prime},\{0, L\}\right)$. Let $\eta \in C_{0}^{\infty}(0,1)$ be such that $\eta \geqslant 0$, and $\int_{0}^{1} \eta(s) \mathrm{d} s=1$. We set $\eta_{h}(s):=\frac{1}{h} \eta\left(\frac{s}{h}\right)$ and we define

$$
\widetilde{R}^{(h)}\left(x_{1}\right):=\int_{-h}^{h} \eta_{h}(s) R^{(h)}\left(x_{1}-s\right) \mathrm{d} s
$$

where we have extended $R^{(h)}$ outside $[0, L]$ by taking $R^{(h)}\left(x_{1}\right)=R^{(h)}(0)$ for every $x_{1}<0, R^{(h)}\left(x_{1}\right)=R^{(h)}(L)$ for every $x_{1}>L$. Clearly $\left|\widetilde{R}^{(h)}\right| \leqslant c$ for every $h$, while properties (2.4) follow by (2.8). Moreover, since by construction

$$
\left|R^{(h)}\left(x_{1}+s\right)-R^{(h)}\left(x_{1}\right)\right|^{2} \leqslant \frac{C}{h} \int_{\Omega} \operatorname{dist}^{2}\left(\nabla_{h} y^{(h)}, \mathrm{SO}(3)\right) \mathrm{d} x \leqslant C h^{3}
$$

for every $|s| \leqslant h$, we have by Jensen inequality that

$$
\left\|\widetilde{R}^{(h)}-R^{(h)}\right\|_{L^{\infty}}^{2} \leqslant C h^{3} .
$$

By the Sobolev-Poincaré inequality and the second inequality in (2.4), there exist constants $Q^{(h)}$ such that $\left\|\widetilde{R}^{(h)}-Q^{(h)}\right\|_{L^{\infty}} \leqslant C h$. Combining this inequality with (2.9), we have that $\left\|R^{(h)}-Q^{(h)}\right\|_{L^{\infty}} \leqslant C h$. This implies that $\operatorname{dist}\left(Q^{(h)}, \mathrm{SO}(3)\right) \leqslant C h$; thus, we may assume that $Q^{(h)}$ belongs to $\mathrm{SO}(3)$ by modifying $Q^{(h)}$ by order $h$, if needed. Now choosing $\bar{R}^{(h)}=Q^{(h)}$ and replacing $R^{(h)}$ by $\left(Q^{(h)}\right)^{\mathrm{T}} R^{(h)}$ and $\widetilde{R}^{(h)}$ by $\left(Q^{(h)}\right)^{\mathrm{T}} \widetilde{R}^{(h)}$, we obtain $(2.5)$.

By a suitable choice of the constants $c^{(h)}$ we may assume

$$
\int_{\Omega}\left(\tilde{y}_{1}^{(h)}-x_{1}\right) \mathrm{d} x=0, \quad \int_{\Omega} \tilde{y}_{k}^{(h)} \mathrm{d} x=0 \quad \text { for } k=2,3 .
$$

Let $A^{(h)}:=\left(R^{(h)}-\mathrm{Id}\right) / h$. By $(2.5)$ there exists $A \in L^{\infty}\left((0, L) ; \mathbb{M}^{3 \times 3}\right)$ such that, up to subsequences,

$$
A^{(h)} \rightarrow A \quad \text { weakly* in } L^{\infty}(0, L) .
$$

On the other hand, it follows from (2.4) that

$$
\frac{\widetilde{R}^{(h)}-\mathrm{Id}}{h} \rightarrow A \quad \text { weakly in } W^{1,2}(0, L) \text {. }
$$

In particular, $A \in W^{1,2}\left((0, L) ; \mathbb{M}^{3 \times 3}\right)$ and $h^{-1}\left(\tilde{R}^{(h)}-I d\right)$ also converges uniformly. Using (2.9) we deduce that

$$
A^{(h)} \rightarrow A \quad \text { uniformly. }
$$

In view of (2.3), this clearly implies the convergence property in (d).

Since $R^{(h)} \in \mathrm{SO}(3)$, we have

$$
A^{(h)}+\left(A^{(h)}\right)^{\mathrm{T}}=-h\left(A^{(h)}\right)^{\mathrm{T}} A^{(h)} .
$$

Hence, $A+A^{\mathrm{T}}=0$. Moreover, after division by $2 h$, we obtain property (e) by (2.12).

Property (b) immediately follows from the convergence in (d) and (2.10). Moreover, $v_{k, 1}=A_{k 1}$ for $k=2,3$, so that $v_{k} \in W^{2,2}(0, L)$, since $A \in W^{1,2}(0, L)$.

The convergence of $\left(u^{(h)}\right)$ follows from (2.3), property (e), and the normalization (2.10).

By the convergence in (d) we deduce that

$$
\frac{(1 / h) \tilde{y}_{2}^{(h)}-x_{2}}{h}-\int_{S} \frac{1}{h^{2}} \tilde{y}_{2}^{(h)} \rightarrow A_{23} x_{3} \quad \text { in } L^{2}(\Omega),
$$


and analogously,

$$
\frac{(1 / h) \tilde{y}_{3}^{(h)}-x_{3}}{h}-\int_{S} \frac{1}{h^{2}} \tilde{y}_{3}^{(h)} \rightarrow-A_{23} x_{2} \quad \text { in } L^{2}(\Omega) .
$$

Now, since $w^{(h)}$ can be written as

$$
\begin{aligned}
w^{(h)}\left(x_{1}\right)= & \frac{1}{\mu(S)} \int_{S} x_{2}\left(\frac{(1 / h) \tilde{y}_{3}^{(h)}-x_{3}}{h}-\int_{S} \frac{1}{h^{2}} \tilde{y}_{3}^{(h)}\right) \mathrm{d} x_{2} \mathrm{~d} x_{3} \\
& -\frac{1}{\mu(S)} \int_{S} x_{3}\left(\frac{(1 / h) \tilde{y}_{2}^{(h)}-x_{2}}{h}-\int_{S} \frac{1}{h^{2}} \tilde{y}_{2}^{(h)}\right) \mathrm{d} x_{2} \mathrm{~d} x_{3},
\end{aligned}
$$

it is clear that $w^{(h)}$ converges to the function $w=-A_{23}=A_{32}$ in $L^{2}(0, L)$. The convergence is actually weak in $W^{1,2}(0, L)$, since one can check that $\left(w_{, 1}^{(h)}\right)$ is bounded in $L^{2}(0, L)$ by $(2.3)$.

By differentiating $\beta_{1}^{(h)}$ with respect to $x_{k}$ with $k=2,3$, we have

$$
\beta_{1, k}^{(h)}=\frac{1}{h^{3}} \tilde{y}_{1, k}^{(h)}+\frac{1}{h} v_{k, 1}^{(h)}=\frac{1}{h^{2}}\left(\frac{1}{h} \tilde{y}_{1, k}^{(h)}+\int_{S} \tilde{y}_{k, 1}^{(h)} \mathrm{d} x_{2} \mathrm{~d} x_{3}\right) .
$$

Note that this can be rewritten as

$$
\beta_{1, k}^{(h)}=\frac{(1 / h) \tilde{y}_{1, k}^{(h)}-R_{1 k}^{(h)}}{h^{2}}+\int_{S} \frac{\tilde{y}_{k, 1}^{(h)}-R_{k 1}^{(h)}}{h^{2}} \mathrm{~d} x_{2} \mathrm{~d} x_{3}+\frac{R_{1 k}^{(h)}+R_{k 1}^{(h)}}{h^{2}},
$$

where the right-hand side is now bounded in $L^{2}(\Omega)$ by virtue of $(2.3)$ and property (e). Therefore, the sequence $\left(\beta_{1, k}^{(h)}\right)$ is bounded in $L^{2}(\Omega)$ for $k=2,3$; using the Poincaré inequality and the fact that $\int_{S} \beta_{1}^{(h)} \mathrm{d} x_{2} \mathrm{~d} x_{3}=0$, we deduce that there exists a constant $C>0$ such that

$$
\int_{S}\left(\beta_{1}^{(h)}(x)\right)^{2} \mathrm{~d} x_{2} \mathrm{~d} x_{3} \leqslant C \int_{S}\left[\left(\beta_{1,2}^{(h)}(x)\right)^{2}+\left(\beta_{1,3}^{(h)}(x)\right)^{2}\right] \mathrm{d} x_{2} \mathrm{~d} x_{3}
$$

for a.e. $x_{1} \in(0, L)$ and for every $h$. Integrating both sides with respect to $x_{1}$, we obtain that the sequence $\left(\beta_{1}^{(h)}\right)$ is bounded in $L^{2}(\Omega)$; so, up to subsequences, $\beta_{1}^{(h)} \rightarrow \beta_{1}$ and $\beta_{1, k}^{(h)} \rightarrow \beta_{1, k}$ weakly in $L^{2}(\Omega)$.

As for the sequences $\left(\beta_{2}^{(h)}\right),\left(\beta_{3}^{(h)}\right)$, we have by differentiation that

$$
\beta_{j, k}^{(h)}=\frac{1}{h^{2}}\left(\frac{1}{h} \tilde{y}_{j, k}^{(h)}-\delta_{j k}-h w^{(h)}\left(1-\delta_{j k}\right)(-1)^{k}\right)
$$

for $j, k=2,3$. Now it is easy to check that

$$
e_{j k}\left(\beta^{(h)}\right):=\frac{1}{2}\left(\beta_{j, k}^{(h)}+\beta_{k, j}^{(h)}\right)=\frac{1}{h^{2}}\left[\operatorname{sym}\left(\nabla_{h} \tilde{y}^{(h)}-\mathrm{Id}\right)\right]_{j k}
$$

for $j, k=2,3$; thus, $\left(e_{j k}\left(\beta^{(h)}\right)\right)$ is bounded in $L^{2}(\Omega)$ by (2.3) and (e). Note that, thanks to the definition of $w^{(h)}$, the function $\left(\beta_{2}^{(h)}\left(x_{1}, \cdot\right), \beta_{3}^{(h)}\left(x_{1}, \cdot\right)\right)$ belongs for a.e. $x_{1} \in(0, L)$ to the closed subspace

$$
\left\{\alpha=\left(\alpha_{2}, \alpha_{3}\right) \in W^{1,2}\left(S ; \mathbb{R}^{2}\right): \int_{S} \alpha \mathrm{d} x_{2} \mathrm{~d} x_{3}=0, \int_{S}\left(x_{3} \alpha_{2}-x_{2} \alpha_{3}\right) \mathrm{d} x_{2} \mathrm{~d} x_{3}=0\right\},
$$


where an inequality of Korn type holds (see [15]). Thus, there exists a constant $C>0$ such that

$$
\left\|\beta_{2}^{(h)}\left(x_{1}, \cdot\right)\right\|_{W^{1,2}(S)}^{2}+\left\|\beta_{3}^{(h)}\left(x_{1}, \cdot\right)\right\|_{W^{1,2}(S)}^{2} \leqslant C \sum_{j, k}\left\|e_{j k}\left(\beta^{(h)}\left(x_{1}, \cdot\right)\right)\right\|_{L^{2}(S)}^{2}
$$

for a.e. $x_{1} \in(0, L)$ and for every $h$. Integrating (2.15) with respect to $x_{1}$, we find that the sequences $\left(\beta_{2}^{(h)}\right),\left(\beta_{3}^{(h)}\right)$ are bounded in $L^{2}(\Omega)$, as well as their derivatives with respect to $x_{2}, x_{3}$. This concludes the proof of (f).

Lemma 2.3. Assume (2.2) is satisfied. Let $R^{(h)}, \tilde{y}^{(h)}, u, A$, and $\beta$ be as in Theorem 2.2. Then

$$
G^{(h)}:=\frac{\left(R^{(h)}\right)^{\mathrm{T}} \nabla_{h} \tilde{y}^{(h)}-\mathrm{Id}}{h^{2}} \rightarrow G \quad \text { in } L^{2}(\Omega),
$$

and the symmetric part of $G$, denoted by $\widetilde{G}$, satisfies

$$
\widetilde{G}=u_{, 1} e_{1} \otimes e_{1}-\frac{A^{2}}{2}+\operatorname{sym}\left(A_{, 1}\left(\begin{array}{c}
0 \\
x_{2} \\
x_{3}
\end{array}\right)\left|\beta_{, 2}\right| \beta_{, 3}\right) .
$$

Moreover,

$$
\liminf _{h \rightarrow 0} \frac{1}{h^{4}} \int_{\Omega} W\left(x, \nabla_{h} y^{(h)}\right) \mathrm{d} x \geqslant \frac{1}{2} \int_{\Omega} Q_{3}(x, \widetilde{G}(x)) \mathrm{d} x,
$$

where $Q_{3}$ is twice the quadratic form of linearized elasticity, i.e.,

$$
Q_{3}(x, F):=\frac{\partial^{2} W}{\partial F^{2}}(x, \mathrm{Id})[F, F] .
$$

Proof. The estimate (2.3) implies that the $L^{2}$-norm of $G^{(h)}$ is bounded; therefore, up to subsequences, there exists $G \in L^{2}\left(\Omega ; \mathbb{M}^{3 \times 3}\right)$ such that $(2.16)$ is satisfied.

In order to identify the symmetric part of $G$ we decompose $R^{(h)} G^{(h)}$ as follows:

$$
R^{(h)} G^{(h)}=\frac{\nabla_{h} \tilde{y}^{(h)}-\mathrm{Id}}{h^{2}}-\frac{R^{(h)}-\mathrm{Id}}{h^{2}},
$$

so that

$$
F^{(h)}:=\operatorname{sym} \frac{\nabla_{h} \tilde{y}^{(h)}-\mathrm{Id}}{h^{2}}=\operatorname{sym}\left(R^{(h)} G^{(h)}\right)+\operatorname{sym} \frac{R^{(h)}-\mathrm{Id}}{h^{2}} .
$$

The right-hand side converges weakly to $\widetilde{G}+A^{2} / 2$ by (2.5), (2.16), and property (e) of Theorem 2.2. Therefore, the sequence $\left(F^{(h)}\right)$ has a weak limit $F$ in $L^{2}(0, L)$, satisfying $F=\widetilde{G}+A^{2} / 2$. To conclude we need only to identify $F$.

Consider the functions

$$
\phi_{1}^{(h)}(x):=\frac{\tilde{y}_{1}^{(h)}(x)-x_{1}}{h^{2}},
$$

which satisfy $\phi_{1,1}^{(h)}=F_{11}^{(h)}$ for every $h$. From property (f) of Theorem 2.2 it follows that the functions $\phi_{1}^{(h)}-u^{(h)}+$ $x_{2} v_{2,1}^{(h)}+x_{3} v_{3,1}^{(h)}$, which are equal to $h \beta_{1}^{(h)}$, converge to 0 strongly in $L^{2}(\Omega)$. Thus, by properties (a) and (b) of Theorem 2.2 we have that

$$
\phi_{1}^{(h)} \rightarrow u-x_{2} v_{2,1}-x_{3} v_{3,1} \quad \text { in } L^{2}(\Omega) .
$$

Now, since $\phi_{1,1}^{(h)}$ converges weakly to $F_{11}$ in $L^{2}(\Omega)$ by construction, we deduce that 


$$
\begin{aligned}
F_{11}(x) & =u_{, 1}\left(x_{1}\right)-x_{2} v_{2,11}\left(x_{1}\right)-x_{3} v_{3,11}\left(x_{1}\right) \\
& =u_{, 1}\left(x_{1}\right)+\sum_{k=2,3} A_{1 k, 1}\left(x_{1}\right) x_{k} .
\end{aligned}
$$

Passing to the limit in the equality (2.14) we immediately have that

$$
e_{j k}(\beta)=F_{j k} \quad \text { for } j, k=2,3 .
$$

It remains to identify $F_{1 k}$ for $k=2,3$. By (2.13) we can write $F_{1 k}^{(h)}$ as follows:

$$
2 F_{1 k}^{(h)}=\frac{\tilde{y}_{k, 1}^{(h)}}{h^{2}}+\frac{\tilde{y}_{1, k}^{(h)}}{h^{3}}=\frac{1}{h^{2}}\left(\tilde{y}_{k, 1}^{(h)}-\int_{S} \tilde{y}_{k, 1}^{(h)} \mathrm{d} x_{2} \mathrm{~d} x_{3}\right)+\beta_{1, k}^{(h)} .
$$

Using the definition of $\beta_{j}$ for $j=2,3$ it is easy to show that

$$
\frac{1}{h^{2}}\left(\tilde{y}_{k, 1}^{(h)}-\int_{S} \tilde{y}_{k, 1}^{(h)} \mathrm{d} x_{2} \mathrm{~d} x_{3}\right)=h \beta_{k, 1}^{(h)}+w_{, 1}^{(h)} x_{k}^{\perp},
$$

hence

$$
2 F_{1 k}^{(h)}=h \beta_{k, 1}^{(h)}+w_{, 1}^{(h)} x_{k}^{\perp}+\beta_{1, k}^{(h)} .
$$

Since the right-hand side converges to $w_{1,} x_{k}^{\perp}+\beta_{1, k}$ weakly in $W^{-1,2}(\Omega)$ by properties (c) and (f) of Theorem 2.2, we have that

$$
2 F_{1 k}=w_{, 1} x_{k}^{\perp}+\beta_{1, k} .
$$

Combining (2.21), (2.22), and (2.23), we obtain (2.17).

We now show the lower bound (2.18). By Taylor expansion we have that

$$
W(x, \operatorname{Id}+A)=\frac{1}{2} \frac{\partial^{2} W}{\partial F^{2}}(x, \operatorname{Id}+t A)[A, A],
$$

where $0<t<1$ depends on $x$ and $A$. We introduce the functions

$$
\chi_{h}(x):= \begin{cases}1 & \text { if } x \in\left\{\left|G^{(h)}\right| \leqslant h^{-1}\right\}, \\ 0 & \text { otherwise. }\end{cases}
$$

Note that from the boundedness of $G^{(h)}$ in $L^{2}(\Omega)$ it follows that $\chi_{h} \rightarrow 1$ in measure. Hence

$$
\chi_{h} G^{(h)} \rightarrow G \text { in } L^{2}(\Omega) .
$$

Using the frame-indifference and (2.24) we obtain

$$
\begin{aligned}
\frac{1}{h^{4}} \int_{\Omega} W\left(x, \nabla_{h} y^{(h)}\right) \mathrm{d} x & \geqslant \frac{1}{h^{4}} \int_{\Omega} \chi_{h} W\left(x, \nabla_{h} y^{(h)}\right) \mathrm{d} x=\frac{1}{h^{4}} \int_{\Omega} \chi_{h} W\left(x,\left(R^{(h)}\right)^{\mathrm{T}} \nabla_{h} y^{(h)}\right) \mathrm{d} x \\
& =\int_{\Omega} \frac{1}{2} \chi_{h} \frac{\partial^{2} W}{\partial F^{2}}\left(x, \operatorname{Id}+h^{2} t_{h}(x) G^{(h)}\right)\left[G^{(h)}, G^{(h)}\right] \mathrm{d} x,
\end{aligned}
$$

where $0<t_{h}(x)<1$. It is convenient to write the last integral as

$$
\begin{aligned}
& \int_{\Omega} \frac{1}{2} \chi_{h} \frac{\partial^{2} W}{\partial F^{2}}\left(x, \operatorname{Id}+h^{2} t_{h}(x) G^{(h)}\right)\left[G^{(h)}, G^{(h)}\right] \mathrm{d} x \\
& \quad=\int_{\Omega} \frac{1}{2}\left(\chi_{h} \frac{\partial^{2} W}{\partial F^{2}}\left(x, \operatorname{Id}+t_{h}(x) h^{2} G^{(h)}\right)\left[G^{(h)}, G^{(h)}\right]-Q_{3}\left(x, \chi_{h} G^{(h)}\right)\right) \mathrm{d} x+\int_{\Omega} \frac{1}{2} Q_{3}\left(x, \chi_{h} G^{(h)}\right) \mathrm{d} x .
\end{aligned}
$$


By Scorza-Dragoni theorem there exists a compact subset $K$ of $\Omega$ such that $\partial^{2} W /\left.\partial F^{2}\right|_{K \times \overline{B_{\delta}}}$ (Id) is continuous (hence, uniformly continuous on compact subsets). Therefore, for every $\varepsilon>0$ we have for $h$ sufficiently small

$$
\begin{aligned}
\int_{\Omega} & \frac{1}{2}\left(\chi_{h} \frac{\partial^{2} W}{\partial F^{2}}\left(x, \operatorname{Id}+t_{h}(x) h^{2} G^{(h)}\right)\left[G^{(h)}, G^{(h)}\right]-Q_{3}\left(x, \chi_{h} G^{(h)}\right)\right) \mathrm{d} x \\
& \geqslant-\frac{1}{2} \varepsilon \int_{K} \chi_{h}\left|G^{(h)}\right|^{2} \mathrm{~d} x \geqslant-C \varepsilon .
\end{aligned}
$$

As for the second integral on the right-hand side of (2.27), it is lower semicontinuous with respect to the convergence (2.25), since $Q_{3}$ is a nonnegative quadratic form. Combining this fact with (2.26), (2.27), and (2.28), we obtain

$$
\liminf _{h \rightarrow 0} \frac{1}{h^{4}} \int_{\Omega} W\left(x, \nabla_{h} y^{(h)}\right) \mathrm{d} x \geqslant \frac{1}{2} \int_{\Omega} Q_{3}(x, G) \mathrm{d} x-C \varepsilon .
$$

Since $\varepsilon$ is arbitrary and $Q_{3}(x, G)$ depends only on the symmetric part of $G$ (by frame-indifference), the thesis follows immediately from (2.29).

\section{Upper bound}

In this section we prove that the lower bound shown in Lemma 2.3 is optimal in the sense specified by the following theorem.

Theorem 3.1. Let $u, w \in W^{1,2}(0, L)$, and $v_{k} \in W^{2,2}(0, L)$ for $k=2,3$. Let $\beta$ be a function in $\mathcal{B}$ (see (2.7)) and let $A \in W^{1,2}\left((0, L) ; \mathbb{M}^{3 \times 3}\right)$ be defined as in (2.6). Set

$$
\widetilde{G}:=u_{, 1} e_{1} \otimes e_{1}-\frac{A^{2}}{2}+\operatorname{sym}\left(A_{, 1}\left(\begin{array}{c}
0 \\
x_{2} \\
x_{3}
\end{array}\right)|\beta, 2| \beta, 3\right) .
$$

Then there exists a sequence $\left(\check{y}^{(h)}\right) \subset W^{1,2}\left(\Omega ; \mathbb{R}^{3}\right)$ such that properties (a)-(f) of Theorem 2.2 are satisfied and

$$
\limsup _{h \rightarrow 0} \frac{1}{h^{4}} \int_{\Omega} W\left(x, \nabla_{h} \check{y}^{(h)}\right) \mathrm{d} x \leqslant \frac{1}{2} \int_{\Omega} Q_{3}(x, \widetilde{G}(x)) \mathrm{d} x,
$$

where $Q_{3}$ is defined as in (2.19).

Proof. Assume first that $u, w, v_{k}, \beta$ are smooth. For every $h>0$ let us consider the function

$$
\check{y}^{(h)}(x):=\left(\begin{array}{c}
x_{1} \\
h x_{2} \\
h x_{3}
\end{array}\right)+\left(\begin{array}{c}
h^{2} u \\
h v_{2} \\
h v_{3}
\end{array}\right)-h^{2}\left(\begin{array}{c}
x_{2} v_{2,1}+x_{3} v_{3,1} \\
x_{3} w \\
-x_{2} w
\end{array}\right)+h^{3} \beta .
$$

Then, properties (a)-(f) are clearly satisfied. Moreover,

$$
\nabla_{h} \check{y}^{(h)}=\mathrm{Id}+\left(\begin{array}{ccc}
h^{2} u_{, 1} & -h v_{2,1} & -h v_{3,1} \\
h v_{2,1} & 0 & -h w \\
h v_{3,1} & h w & 0
\end{array}\right)-h^{2}\left(\begin{array}{c|c}
x_{2} v_{2,11}+x_{3} v_{3,11} \\
x_{3} w_{, 1} \\
-x_{2} w_{, 1}
\end{array}\left|\beta_{, 2}\right| \beta_{, 3}\right)+\mathrm{O}\left(h^{3}\right) .
$$

Using the identity $\left(\mathrm{Id}+B^{\mathrm{T}}\right)(\mathrm{Id}+B)=\operatorname{Id}+2 \operatorname{sym} B+B^{\mathrm{T}} B$, we obtain for the nonlinear strain

$$
\left(\nabla_{h} \check{y}^{(h)}\right)^{\mathrm{T}} \nabla_{h} \check{y}^{(h)}=\mathrm{Id}+2 h^{2} u_{, 1} e_{1} \otimes e_{1}+2 h^{2} \operatorname{sym}\left(A_{, 1}\left(\begin{array}{c}
0 \\
x_{2} \\
x_{3}
\end{array}\right)|\beta, 2| \beta, 3\right)+h^{2} A^{\mathrm{T}} A+\mathrm{O}\left(h^{3}\right) .
$$


Taking the square root and using the definition of $\widetilde{G}$, we have that

$$
\left[\left(\nabla_{h} \check{y}^{(h)}\right)^{\mathrm{T}} \nabla_{h} \check{y}^{(h)}\right]^{1 / 2}=\mathrm{Id}+h^{2} \widetilde{G}+\mathrm{O}\left(h^{3}\right) .
$$

We have det $\nabla_{h} \check{y}^{(h)}>0$ for sufficiently small $h$. Hence by frame-indifference

$$
W\left(x, \nabla_{h} \check{y}^{(h)}\right)=W\left(x,\left[\left(\nabla_{h} \check{y}^{(h)}\right)^{\mathrm{T}} \nabla_{h} \check{y}^{(h)}\right]^{1 / 2}\right) ;
$$

thus, by (3.3) and Taylor expansion, we obtain

$$
\frac{1}{h^{4}} W\left(x, \nabla_{h} \check{y}^{(h)}\right) \rightarrow \frac{1}{2} Q_{3}(x, \widetilde{G}) \text { a.e., }
$$

and

$$
\frac{1}{h^{4}} W\left(x, \nabla_{h} \check{y}^{(h)}\right) \leqslant \frac{1}{2} \gamma|\widetilde{G}|^{2}+C h \leqslant C\left(|A|^{4}+\left|A_{, 1}\right|^{2}+\left|\beta_{, 2}\right|^{2}+\left|\beta_{, 3}\right|^{2}+\left|u_{, 1}\right|^{2}+1\right) \in L^{1}(\Omega) .
$$

Now the inequality (3.1) follows by the dominated convergence theorem.

In the general case, it is enough to smoothly approximate $u, w$ in the strong topology of $W^{1,2}, v_{k}$ in the strong topology of $W^{2,2}$, and $\beta, \beta_{, k}$ in the strong topology of $L^{2}$, and to use the continuity of the right-hand side of (3.1) with respect to these convergences.

\section{Identification of the $\Gamma$-limit}

Let $Q:(0, L) \times \mathbb{R} \times \mathbb{M}_{\text {skew }}^{3 \times 3} \rightarrow[0,+\infty)$ be defined as

$$
Q\left(x_{1}, t, F\right):=\min _{\alpha \in W^{1,2}\left(S ; \mathbb{R}^{3}\right)} \int_{S} Q_{3}\left(x,\left(F\left(\begin{array}{c}
0 \\
x_{2} \\
x_{3}
\end{array}\right)+t e_{1}\left|\alpha_{, 2}\right| \alpha_{, 3}\right)\right) \mathrm{d} x_{2} \mathrm{~d} x_{3},
$$

where $Q_{3}$ is the quadratic form defined in (2.19). Physically the minimizer $\alpha$ in (4.1) corresponds to the warping of cross-section, induced by the bending and torsion encoded in $F$ and the stretch $t$ in the direction of the rod.

For $u, w \in W^{1,2}(0, L)$ and $v_{2}, v_{3} \in W^{2,2}(0, L)$ we introduce the functional

$$
I^{0}\left(u, v_{2}, v_{3}, w\right):=\frac{1}{2} \int_{0}^{L} Q\left(x_{1}, u_{, 1}+\frac{1}{2}\left(v_{2,1}^{2}+v_{3,1}^{2}\right), A_{, 1}\right) \mathrm{d} x_{1},
$$

where $A \in W^{1,2}\left((0, L) ; \mathbb{M}^{3 \times 3}\right)$ denotes the matrix

$$
A:=\left(\begin{array}{ccc}
0 & -v_{2,1} & -v_{3,1} \\
v_{2,1} & 0 & -w \\
v_{3,1} & w & 0
\end{array}\right)
$$

The main result of this section is the proof of the $\Gamma$-convergence of the functionals $\left(1 / h^{4}\right) I^{(h)}$ to $I^{0}$. Before stating the theorem we analyse some properties of the limit density $Q$.

Remark 4.1. The minimum in (4.1) is attained. To prove this, recall first that $Q_{3}(x, G)$ depends only on the symmetric part of $G$. Thus the functional in (4.1) is invariant under the transformation $\alpha \mapsto \alpha+c_{1}+c_{2} x^{\perp}$, and hence the minimum can be computed on the subspace

$$
V:=\left\{\alpha \in W^{1,2}\left(S ; \mathbb{R}^{3}\right): \int_{S} \alpha \mathrm{d} x_{2} \mathrm{~d} x_{3}=0, \int_{S}\left(x_{3} \alpha_{2}-x_{2} \alpha_{3}\right) \mathrm{d} x_{2} \mathrm{~d} x_{3}=0\right\} .
$$


Since $Q_{3}(x, F) \geqslant C|\operatorname{sym} F|^{2}$ for every $F$, the minimizing sequences contained in $V$ are compact with respect to the weak topology of $W^{1,2}\left(S ; \mathbb{R}^{3}\right)$ (using again Korn's inequality for $\left(\alpha_{2}, \alpha_{3}\right)$, see, e.g., [15]). Moreover, the functional to minimize is lower semicontinuous in $\alpha$ with respect to this convergence. This is enough to guarantee the existence of a minimizer. The strict convexity of $Q_{3}(x, \cdot)$ on the set of symmetric matrices ensures also that the minimizer is unique in $V$.

Remark 4.2 (Euler-Lagrange equation). Fix $x_{1} \in(0, L), t \in \mathbb{R}$, and $F \in \mathbb{M}_{\text {skew }}^{3 \times 3}$. Let $\alpha^{\text {min }} \in V$ be the minimizer of the problem (4.1). For sake of notation we set

$$
g\left(x_{2}, x_{3}\right):=F\left(\begin{array}{c}
0 \\
x_{2} \\
x_{3}
\end{array}\right)+t e_{1}, \quad b_{i j}^{h k}(x):=\frac{\partial^{2} W}{\partial F_{i h} \partial F_{j k}}(x, \mathrm{Id}),
$$

and we call $B^{h k}$ the matrix in $\mathbb{M}^{3 \times 3}$, whose elements are given by $\left(B^{h k}\right)_{i j}=b_{i j}^{h k}$. Then $\alpha^{\text {min }}$ satisfies the following Euler-Lagrange equation:

$$
\int_{S} \sum_{h, k=2,3}\left(B^{h k} \alpha_{, k}^{\min }, \varphi_{, h}\right) \mathrm{d} x_{2} \mathrm{~d} x_{3}=-\int_{S} \sum_{h=2,3}\left(B^{h 1} g, \varphi, h\right) \mathrm{d} x_{2} \mathrm{~d} x_{3}
$$

for every $\varphi \in W^{1,2}\left(S ; \mathbb{R}^{3}\right)$.

From this equation it is clear that $\alpha^{\min }$ depends linearly on the pair $(t, F)$. Hence $Q$ is a quadratic form of $(t, F)$. Moreover, $Q$ is uniformly positive definite, i.e.,

$$
Q\left(x_{1}, t, F\right) \geqslant \widetilde{C}\left(t^{2}+|F|^{2}\right) \quad \forall t \in \mathbb{R}, \forall F \in \mathbb{M}_{\text {skew }}^{3 \times 3},
$$

where the constant $\widetilde{C}$ does not depend on $x_{1}$. To see this note that $Q_{3}(x, G) \geqslant C|\operatorname{sym} G|^{2}$ by hypothesis (iv) on $W$. Thus it suffices to establish the bound (4.4) for the special quadratic form $Q_{3}(x, G)=|\operatorname{sym} G|^{2}$. If it failed, there would exist $(t, F) \neq(0,0)$ and $\alpha=\alpha^{\min } \in V$ such that

$$
\operatorname{sym}\left(F\left(\begin{array}{c}
0 \\
x_{2} \\
x_{3}
\end{array}\right)+t e_{1}\left|\alpha_{, 2}\right| \alpha_{, 3}\right)=0 .
$$

The equations for the $1 k$ component yield

$$
\begin{aligned}
& F_{12} x_{2}+F_{13} x_{3}+t=0, \\
& F_{23} x_{3}+\alpha_{1,2}=0, \\
& -F_{23} x_{2}+\alpha_{1,3}=0 .
\end{aligned}
$$

Thus $F_{12}=F_{13}=t=0$, and by derivation of the two last identities we deduce $F_{23}=0$, a contradiction.

Remark 4.3. For future reference we note that there exists a constant $C^{\prime}$ (independent of $x_{1}, t$, and $F$ ) such that

$$
\left\|\alpha_{, 2}^{\min }\right\|_{L^{2}(S)}^{2}+\left\|\alpha_{, 3}^{\min }\right\|_{L^{2}(S)}^{2} \leqslant C^{\prime}\|g\|_{L^{2}(S)}^{2} .
$$

Indeed, since $Q_{3}(x, F) \geqslant C|\operatorname{sym} F|^{2}$ for every matrix $F$, we have

$$
\frac{1}{C} \sum_{h, k=2,3}\left(B^{h k} \varphi_{, k}, \varphi_{, h}\right) \geqslant \sum_{k=2,3}\left|\varphi_{1, k}\right|^{2}+\sum_{j, k=2,3}\left|e_{j k}(\varphi)\right|^{2} \quad \forall \varphi \in W^{1,2}\left(S ; \mathbb{R}^{3}\right),
$$

where $2 e_{j k}(\varphi):=\varphi_{j, k}+\varphi_{k, j}$. Taking $\alpha^{\text {min }}$ as test function in (4.3) and using the above inequality, we obtain 


$$
\begin{aligned}
\int_{S} \sum_{k=2,3}\left|\alpha_{1, k}^{\min }\right|^{2} \mathrm{~d} x_{2} \mathrm{~d} x_{3}+\int_{S} \sum_{j, k=2,3}\left|e_{j k}\left(\alpha^{\min }\right)\right|^{2} \mathrm{~d} x_{2} \mathrm{~d} x_{3} & \leqslant-\frac{1}{C} \int_{S} \sum_{h=2,3}\left(B^{h 1} g, \alpha_{, h}^{\min }\right) \mathrm{d} x_{2} \mathrm{~d} x_{3} \\
& \leqslant \frac{1}{C} \sum_{h=2,3}\left\|B^{h 1} g\right\|_{L^{2}(S)}\left\|\alpha_{, h}^{\min }\right\|_{L^{2}(S)} .
\end{aligned}
$$

By Korn inequality there exists a constant $C_{1}>0$ such that

$$
\int_{S} \sum_{j, k=2,3}\left|\alpha_{j, k}^{\min }\right|^{2} \mathrm{~d} x_{2} \mathrm{~d} x_{3} \leqslant C_{1} \int_{S} \sum_{j, k=2,3}\left|e_{j k}\left(\alpha^{\min }\right)\right|^{2} \mathrm{~d} x_{2} \mathrm{~d} x_{3} ;
$$

hence, by (4.6) we have

$$
\begin{aligned}
\left\|\alpha_{, 2}^{\min }\right\|_{L^{2}(S)}^{2}+\left\|\alpha_{, 3}^{\min }\right\|_{L^{2}(S)}^{2} & \leqslant C_{2} \sum_{h=2,3}\left\|B^{h 1} g\right\|_{L^{2}(S)}\left\|\alpha_{, h}^{\min }\right\|_{L^{2}(S)} \\
& \leqslant C_{3}\|g\|_{L^{2}(S)} \sum_{h=2,3}\left\|\alpha_{, h}^{\min }\right\|_{L^{2}(S)},
\end{aligned}
$$

where the last inequality follows from the boundedness of the entries of $B^{h k}$ (this is a consequence of the assumption (ii) on $W$ ). Inequality (4.5) follows immediately from (4.7).

Remark 4.4. When $Q_{3}$ does not depend on $x_{2}$ and $x_{3}$, we can find a more explicit representation for $Q$. More precisely, the form $Q$ can be decomposed into the sum of two quadratic forms

$$
Q\left(x_{1}, t, F\right)=Q_{1}\left(x_{1}, t\right)+Q_{2}\left(x_{1}, F\right)
$$

where

$$
\begin{aligned}
& Q_{1}\left(x_{1}, t\right):=\min _{a, b \in \mathbb{R}^{3}} Q_{3}\left(x_{1},\left(t e_{1}|a| b\right)\right), \\
& Q_{2}\left(x_{1}, F\right):=Q\left(x_{1}, 0, F\right) .
\end{aligned}
$$

To see this fix $x_{1}, t$, and $F$, and let $\alpha \in W^{1,2}\left(S ; \mathbb{R}^{3}\right)$. It is convenient to introduce the following quantities:

$$
\begin{aligned}
& a:=\int_{S} \alpha_{, 2} \mathrm{~d} x_{2} \mathrm{~d} x_{3}, \quad b:=\int_{S} \alpha_{, 3} \mathrm{~d} x_{2} \mathrm{~d} x_{3}, \\
& \beta\left(x_{2}, x_{3}\right):=\alpha\left(x_{2}, x_{3}\right)-x_{2} a-x_{3} b .
\end{aligned}
$$

By expanding the quadratic form $Q_{3}$ we have that

$$
\begin{aligned}
& \int_{S} Q_{3}\left(x_{1},\left(F\left(\begin{array}{c}
0 \\
x_{2} \\
x_{3}
\end{array}\right)+t e_{1}\left|\alpha_{, 2}\right| \alpha_{, 3}\right)\right) \mathrm{d} x_{2} \mathrm{~d} x_{3} \\
& =Q_{3}\left(x_{1},\left(t e_{1}|a| b\right)\right)+\int_{S} Q_{3}\left(x_{1},\left(F\left(\begin{array}{c}
0 \\
x_{2} \\
x_{3}
\end{array}\right)\left|\beta_{, 2}\right| \beta_{, 3}\right)\right) \mathrm{d} x_{2} \mathrm{~d} x_{3} .
\end{aligned}
$$

The absence of a coupling term is due to the fact that the matrix $\left(t e_{1}|a| b\right)$ is independent of $x_{2}, x_{3}$, while the matrix

$$
\left(F\left(\begin{array}{c}
0 \\
x_{2} \\
x_{3}
\end{array}\right)\left|\beta_{, 2}\right| \beta_{, 3}\right)
$$

has zero average on $S$ by (2.1) and by the definition of $\beta$. Now, equality (4.10) implies that $Q\left(x_{1}, t, F\right) \geqslant$ $Q_{1}\left(x_{1}, t\right)+Q_{2}\left(x_{1}, F\right)$. 
Vice-versa, let $\beta \in W^{1,2}\left(S ; \mathbb{R}^{3}\right)$ be a minimizer for the problem defining $Q\left(x_{1}, 0, F\right)$. Then expanding the quadratic form $Q_{3}$ and using the fact that $Q_{3}$ is nonnegative, it is possible to show that $\beta$ must satisfy

$$
\int_{S} \beta_{, 2} \mathrm{~d} x_{2} \mathrm{~d} x_{3}=\int_{S} \beta_{, 3} \mathrm{~d} x_{2} \mathrm{~d} x_{3}=0 .
$$

Let $(a, b) \in \mathbb{R}^{3} \times \mathbb{R}^{3}$ be a minimizer for (4.8) and let

$$
\alpha\left(x_{2}, x_{3}\right):=\beta\left(x_{2}, x_{3}\right)+x_{2} a+x_{3} b .
$$

The identity (4.10) now implies the required inequality.

The formula (4.9) can be further simplified if the stored energy function is isotropic or if $S$ is a circle (see Remarks 3.5 and 3.6 in [12]).

We now state and prove the convergence result.

Theorem 4.5. As $h \rightarrow 0$, the functionals $\left(1 / h^{4}\right) I^{(h)}$ are $\Gamma$-convergent to the functional $I^{0}$ given in (4.2), in the following sense:

(i) (compactness and liminf inequality) if $\limsup _{h \rightarrow 0} h^{-4} I^{(h)}\left(y^{(h)}\right)<+\infty$, then there exist constants $\bar{R}^{(h)} \in$ $\mathrm{SO}(3)$ and $c^{(h)} \in \mathbb{R}$ such that (up to subsequences) $\bar{R}^{(h)} \rightarrow \bar{R}$ and the functions defined by

$$
\begin{aligned}
\tilde{y}^{(h)}(x) & :=\left(\bar{R}^{(h)}\right)^{\mathrm{T}} y^{(h)}(x)-c^{(h)}, \quad u^{(h)}\left(x_{1}\right):=\int_{S} \frac{\tilde{y}_{1}^{(h)}(x)-x_{1}}{h^{2}} \mathrm{~d} x_{2} \mathrm{~d} x_{3}, \\
v_{k}^{(h)}\left(x_{1}\right) & :=\int_{S} \frac{\tilde{y}_{k}^{(h)}(x)}{h} \mathrm{~d} x_{2} \mathrm{~d} x_{3} \quad \text { for } k=2,3, \\
w^{(h)}\left(x_{1}\right) & :=\frac{1}{\mu(S)} \int_{S} \frac{x_{2} \tilde{y}_{3}^{(h)}(x)-x_{3} \tilde{y}_{2}^{(h)}(x)}{h^{2}} \mathrm{~d} x_{2} \mathrm{~d} x_{3},
\end{aligned}
$$

satisfy

(1) $\nabla_{h} \tilde{y}^{(h)} \rightarrow \operatorname{Id}$ in $L^{2}(\Omega)$

(2) there exist $u, w \in W^{1,2}(0, L)$ such that $u^{(h)} \rightarrow u$ and $w^{(h)} \rightarrow w$ weakly in $W^{1,2}(0, L)$;

(3) there exist $v_{k} \in W^{2,2}(0, L)$ such that $v_{k}^{(h)} \rightarrow v_{k}$ strongly in $W^{1,2}(0, L)$ for $k=2,3$.

Moreover, we have

$$
\liminf _{h \rightarrow 0} \frac{1}{h^{4}} I^{(h)}\left(y^{(h)}\right) \geqslant I^{0}\left(u, v_{2}, v_{3}, w\right)
$$

(ii) (limsup inequality) for every $u, w \in W^{1,2}(0, L), v_{2}, v_{3} \in W^{2,2}(0, L)$ there exists $\left(\check{y}^{(h)}\right)$ such that (1)-(3) hold (with $\tilde{y}^{(h)}$ replaced by $\check{y}^{(h)}$ ) and

$$
\limsup _{h \rightarrow 0} \frac{1}{h^{4}} I^{(h)}\left(\check{y}^{(h)}\right) \leqslant I^{0}\left(u, v_{2}, v_{3}, w\right) .
$$

Proof. (i) Properties (1)-(3) follow from Theorem 2.2. By Lemma 2.3 we know that

$$
\liminf _{h \rightarrow 0} \frac{1}{h^{4}} \int_{\Omega} W\left(x_{1}, \nabla_{h} y^{(h)}\right) \mathrm{d} x \geqslant \frac{1}{2} \int_{\Omega} Q_{3}(x, \widetilde{G}(x)) \mathrm{d} x,
$$

where $\widetilde{G}$ can be written as 


$$
\begin{aligned}
\widetilde{G}(x)= & u_{, 1} e_{1} \otimes e_{1}-\frac{A^{2}}{2}+\operatorname{sym}\left(A_{, 1}\left(\begin{array}{c}
0 \\
x_{2} \\
x_{3}
\end{array}\right)\left|\beta_{, 2}\right| \beta_{, 3}\right) \\
= & \operatorname{sym}\left(A_{, 1}\left(\begin{array}{c}
0 \\
x_{2} \\
x_{3}
\end{array}\right)+\left(u_{, 1}+\frac{1}{2}\left(v_{2,1}^{2}+v_{3,1}^{2}\right)\right) e_{1}\left|\beta_{, 2}\right| \beta_{, 3}\right) \\
& +\frac{1}{2}\left(\begin{array}{ccc}
0 & v_{3,1} w & -v_{2,1} w \\
v_{3,1} w & w^{2}+v_{2,1}^{2} & v_{2,1} v_{3,1} \\
-v_{2,1} w & v_{2,1} v_{3,1} & w^{2}+v_{3,1}^{2}
\end{array}\right) .
\end{aligned}
$$

Set $\alpha(x):=\beta(x)+\frac{1}{2} x_{2} \gamma_{2}\left(x_{1}\right)+\frac{1}{2} x_{3} \gamma_{3}\left(x_{1}\right)$, where

$$
\begin{aligned}
& \gamma_{2}\left(x_{1}\right):=2 v_{3,1} w e_{1}+\left(w^{2}+v_{2,1}^{2}\right) e_{2}+v_{2,1} v_{3,1} e_{3}, \\
& \gamma_{3}\left(x_{1}\right):=-2 v_{2,1} w e_{1}+v_{2,1} v_{3,1} e_{2}+\left(w^{2}+v_{3,1}^{2}\right) e_{3} .
\end{aligned}
$$

Using these new definitions, we have that

$$
\widetilde{G}(x)=\operatorname{sym}\left(A_{, 1}\left(\begin{array}{c}
0 \\
x_{2} \\
x_{3}
\end{array}\right)+\left(u_{, 1}+\frac{1}{2}\left(v_{2,1}^{2}+v_{3,1}^{2}\right)\right) e_{1}\left|\alpha_{, 2}\right| \alpha_{, 3}\right) .
$$

Since $\alpha\left(x_{1}, \cdot\right) \in W^{1,2}\left(S ; \mathbb{R}^{3}\right)$ for a.e. $x_{1} \in(0, L)$, it follows from the definition of $Q$ that

$$
\int_{\Omega} Q_{3}(x, \widetilde{G}(x)) \mathrm{d} x \geqslant \int_{0}^{L} Q\left(x_{1}, u_{, 1}+\frac{1}{2}\left(v_{2,1}^{2}+v_{3,1}^{2}\right), A_{, 1}\right) \mathrm{d} x_{1} .
$$

The thesis (4.11) now simply follows from (4.12) and (4.17).

(ii) Let $u, w \in W^{1,2}(0, L)$ and $v_{2}, v_{3} \in W^{2,2}(0, L)$. Let $\alpha\left(x_{1}, \cdot\right) \in V$ be the solution of the minimum problem defining $Q\left(x_{1}, u_{, 1}+\frac{1}{2}\left(v_{2,1}^{2}+v_{3,1}^{2}\right), A_{, 1}\right)$ (see Remark 4.1 for the definition of the space $V$ ). Then $\alpha$ and its derivatives $\alpha_{, 2}, \alpha, 3$ belong to $L^{2}\left(\Omega ; \mathbb{R}^{3}\right)$. Indeed, by the Sobolev-Poincaré inequality we have

$$
\left\|\alpha\left(x_{1}, \cdot\right)\right\|_{L^{2}(S)}^{2} \leqslant C\left(\left\|\alpha_{, 2}\left(x_{1}, \cdot\right)\right\|_{L^{2}(S)}^{2}+\left\|\alpha, 3\left(x_{1}, \cdot\right)\right\|_{L^{2}(S)}^{2}\right)
$$

for a.e. $x_{1} \in(0, L)$; thus, integrating with respect to $x_{1}$, we deduce

$$
\|\alpha\|_{L^{2}(\Omega)}^{2} \leqslant C\left(\|\alpha, 2\|_{L^{2}(\Omega)}^{2}+\|\alpha, 3\|_{L^{2}(\Omega)}^{2}\right) .
$$

Therefore it is enough to prove that $\alpha_{, 2}, \alpha_{, 3} \in L^{2}\left(\Omega ; \mathbb{R}^{3}\right)$. This can be done by integrating the estimate (4.5) with respect to $x_{1}$; in that way, we obtain the following inequality:

$$
\left\|\alpha_{, 2}\right\|_{L^{2}(\Omega)}^{2}+\left\|\alpha_{, 3}\right\|_{L^{2}(\Omega)}^{2} \leqslant C^{\prime}\left(\left\|A_{, 1}\right\|_{L^{2}(0, L)}^{2}+\left\|u_{, 1}\right\|_{L^{2}(0, L)}^{2}+\sum_{k=2,3}\left\|v_{k, 1}\right\|_{L^{4}(0, L)}^{4}\right) .
$$

The right-hand side is bounded, since $A \in W^{1,2}\left((0, L) ; \mathbb{M}^{3 \times 3}\right), u \in W^{1,2}(0, L)$, and $v_{k, 1}=A_{k 1} \in L^{\infty}(0, L)$ by the Sobolev embedding theorem.

Now let $\gamma_{2}, \gamma_{3}$ be defined as in (4.14), (4.15). We denote by $\tilde{\alpha}$ the function given by

$$
\tilde{\alpha}(x):=\alpha(x)-\omega\left(x_{1}\right) x^{\perp},
$$

where $\omega$ is chosen in such a way that the function

$$
\beta(x):=\tilde{\alpha}(x)-\frac{1}{2} x_{2} \gamma_{2}\left(x_{1}\right)-\frac{1}{2} x_{3} \gamma_{3}\left(x_{1}\right)
$$


belongs to the set $\mathcal{B}$ defined in (2.7). Since $Q_{3}\left(x_{1}, F\right)=Q_{3}\left(x_{1}, \operatorname{sym} F\right)$, it is clear that $\tilde{\alpha}\left(x_{1}, \cdot\right)$ is still a minimizer for the problem defining $Q\left(x_{1}, u_{, 1}+\frac{1}{2}\left(v_{2,1}^{2}+v_{3,1}^{2}\right), A_{, 1}\right)$. To conclude it is enough to use the equivalence of (4.16) and (4.13) and to apply Theorem 3.1 to the functions $u, w, v_{k}$, and $\beta$.

\section{Asymptotic behaviour of solutions}

In Theorem 2.2 we have shown that sequences whose energy $\left(1 / h^{4}\right) I^{(h)}$ is finite, converge strongly in $W^{1,2}(\Omega)$ to a rigid motion. The aim of this section is to characterise the asymptotic behaviour of the deviation of solutions from the rigid motion and of the nonlinear strain from the identity. We will then compare this result with the expansion obtained by Murat and Sili in the setting of linear elasticity [13].

Theorem 5.1. Let $\left(y^{(h)}\right)$ be a sequence in $W^{1,2}\left(\Omega ; \mathbb{R}^{3}\right)$ such that

$$
\limsup _{h \rightarrow 0} \frac{1}{h^{4}} \int_{\Omega} W\left(x, \nabla_{h} y^{(h)}\right) \mathrm{d} x<+\infty .
$$

Let $\bar{R}^{(h)} \in \mathrm{SO}(3)$ and $c^{(h)} \in \mathbb{R}$ be as in Theorem 2.2 and let $\tilde{y}^{(h)}:=\left(\bar{R}^{(h)}\right)^{\mathrm{T}} y^{(h)}-c^{(h)}$. Then there exist $u, w \in W^{1,2}(0, L), v_{2}, v_{3} \in W^{2,2}(0, L)$, and $\beta \in \mathcal{B}$ (see (2.7) for the definition of $\left.\mathcal{B}\right)$ such that the scaled deviations of $\tilde{y}^{(h)}$ from the identity satisfy, up to subsequences,

$$
\begin{aligned}
\phi_{1}^{(h)} & :=\frac{\tilde{y}_{1}^{(h)}-x_{1}}{h^{2}} \rightarrow u-x_{2} v_{2,1}-x_{3} v_{3,1} \quad \text { weakly in } W^{1,2}(\Omega), \\
\phi_{k}^{(h)} & :=\frac{\tilde{y}_{k}^{(h)}-h x_{k}}{h} \rightarrow v_{k} \quad \text { strongly in } W^{1,2}(\Omega),
\end{aligned}
$$

while the scaled nonlinear strain satifies

$$
\frac{\left[\left(\nabla_{h} y^{(h)}\right)^{\mathrm{T}} \nabla_{h} y^{(h)}\right]^{1 / 2}-\mathrm{Id}}{h^{2}} \rightarrow \widetilde{G} \quad \text { weakly in } L^{2}(\Omega),
$$

where

$$
\widetilde{G}=u_{, 1} e_{1} \otimes e_{1}-\frac{A^{2}}{2}+\operatorname{sym}\left(A_{, 1}\left(\begin{array}{c}
0 \\
x_{2} \\
x_{3}
\end{array}\right)\left|\beta_{, 2}\right| \beta_{, 3}\right)
$$

and $A$ is defined as in (2.6).

If we assume in addition that

$$
\lim _{h \rightarrow 0} \frac{1}{h^{4}} \int_{\Omega} W\left(x, \nabla_{h} y^{(h)}\right) \mathrm{d} x=\frac{1}{2} \int_{0}^{L} Q\left(x_{1}, u_{, 1}+\frac{1}{2}\left(v_{2,1}^{2}+v_{3,1}^{2}\right), A_{, 1}\right) \mathrm{d} x_{1},
$$

then the convergence in (5.1) and (5.3) are strong. Furthermore, the matrix $\widetilde{G}$ satisfies

$$
\widetilde{G}=u_{, 1} e_{1} \otimes e_{1}+\operatorname{sym}\left(A_{, 1}\left(\begin{array}{c}
0 \\
x_{2} \\
x_{3}
\end{array}\right)\left|\alpha_{, 2}^{\min }\right| \alpha_{, 3}^{\min }\right)
$$

where $\alpha^{\min }$ is the solution in $V$ of the minimum problem defining $Q\left(x_{1}, u_{, 1}+\frac{1}{2}\left(v_{2,1}^{2}+v_{3,1}^{2}\right), A_{, 1}\right)$.

Proof. Properties (5.1) and (5.2) are consequences of Theorem 2.2. Indeed, from (2.3), (e), and (d) it follows that the sequence $\left(\nabla \phi_{1}^{(h)}\right)$ is bounded in $L^{2}(\Omega)$. Since $\phi_{1}^{(h)} \rightarrow u-x_{2} v_{2,1}-x_{3} v_{3,1}$ strongly in $L^{2}(\Omega)$ by $(2.20)$, we 
have that (5.1) holds up to subsequences. From (d) it follows that $\nabla \phi_{k}^{(h)} \rightarrow v_{k, 1} e_{1}$ strongly in $L^{2}(\Omega)$. By $(2.10)$ and the Poincaré inequality we conclude that (5.2) holds true.

The convergence in (5.3) easily follows from (2.16).

Assume now that (5.4) holds. Combining it with (2.26), (2.27), and (2.28), we obtain

$$
\begin{aligned}
& \frac{1}{2} \int_{0}^{L} Q\left(x_{1}, u_{, 1}+\frac{1}{2}\left(v_{2,1}^{2}+v_{3,1}^{2}\right), A, 1\right) \mathrm{d} x_{1} \\
& \quad=\limsup _{h \rightarrow 0} \frac{1}{h^{4}} \int_{\Omega} W\left(x, \nabla_{h} y^{(h)}\right) \mathrm{d} x \geqslant \underset{h \rightarrow 0}{\limsup _{\Omega}} \frac{1}{h^{4}} \int_{\Omega} \chi_{h} W\left(x, \nabla_{h} y^{(h)}\right) \mathrm{d} x \\
& \quad \geqslant \limsup _{h \rightarrow 0} \frac{1}{2} \int_{\Omega} Q_{3}\left(x, \chi_{h} G^{(h)}\right) \mathrm{d} x \geqslant \frac{1}{2} \int_{\Omega} Q_{3}(x, \widetilde{G}(x)) \mathrm{d} x .
\end{aligned}
$$

Since the last term is always greater or equal than the first one, all the inequalities above are equalities and for a.e. $x_{1} \in(0, L)$

$$
Q\left(x_{1}, u_{, 1}+\frac{1}{2}\left(v_{2,1}^{2}+v_{3,1}^{2}\right), A_{, 1}\right)=\int_{S} Q_{3}(x, \widetilde{G}(x)) \mathrm{d} x_{2} \mathrm{~d} x_{3} .
$$

As proved in Remark 4.1, the minimum problem defining $Q$ has a unique solution $\alpha^{\min }$ in the subspace $V$, so that, writing $\widetilde{G}$ as in (4.16),

$$
\alpha^{\min }(x)=\alpha(x)-\omega\left(x_{1}\right) x^{\perp}
$$

where

$$
\alpha(x)=\beta(x)+\frac{1}{2} x_{2} \gamma_{2}\left(x_{1}\right)+\frac{1}{2} x_{3} \gamma_{3}\left(x_{1}\right),
$$

$\gamma_{2}, \gamma_{3}$ are defined as in (4.14) and (4.15), and $\omega$ is uniquely determined by the requirement that $\alpha-\omega x^{\perp} \in V$. Substituting (5.7) in the expression of $\widetilde{G}$ we obtain (5.5).

Next, using the coerciveness of $Q_{3}$, from (2.25) and the fact that the equality holds in the last inequality of (5.6) we can deduce that

$$
\chi_{h} \operatorname{sym} G^{(h)} \rightarrow \widetilde{G} \quad \text { strongly in } L^{2}(\Omega) .
$$

By the definition of $G^{(h)}$ we obtain

$$
\left(\nabla_{h} y^{(h)}\right)^{\mathrm{T}} \nabla_{h} y^{(h)}=\mathrm{Id}+2 h^{2} \operatorname{sym} G^{(h)}+h^{4}\left(G^{(h)}\right)^{\mathrm{T}} G^{(h)},
$$

so that we have the following bound

$$
\left|\left[\left(\nabla_{h} y^{(h)}\right)^{\mathrm{T}} \nabla_{h} y^{(h)}\right]^{1 / 2}-\left(\operatorname{Id}+h^{2} \operatorname{sym} G^{(h)}\right)\right| \leqslant C h^{4}\left|G^{(h)}\right|^{2} .
$$

Multiplying both sides by $\left(1 / h^{2}\right) \chi_{h}$ and using the fact that $h^{2}\left|G^{(h)}\right| \leqslant h$ on the set $\left\{x \in \Omega: \chi_{h}(x) \neq 0\right\}$, we get

$$
\chi_{h} \frac{\left[\left(\nabla_{h} y^{(h)}\right)^{\mathrm{T}} \nabla_{h} y^{(h)}\right]^{1 / 2}-\mathrm{Id}}{h^{2}} \rightarrow \widetilde{G} \quad \text { strongly in } L^{2}(\Omega) .
$$

Since the equality holds in the first inequality of (5.6), we have that

$$
\lim _{h \rightarrow 0} \frac{1}{h^{4}} \int_{\Omega}\left(1-\chi_{h}\right) W\left(x, \nabla_{h} y^{(h)}\right) \mathrm{d} x=0 .
$$


Using the inequality

$$
\left|\left(A^{\mathrm{T}} A\right)^{1 / 2}-\mathrm{Id}\right|^{2} \leqslant \operatorname{dist}^{2}(A, \operatorname{SO}(3)) \leqslant \frac{1}{C} W(x, A) \quad \forall A \in \mathbb{M}^{3 \times 3},
$$

we have by (5.10)

$$
\limsup _{h \rightarrow 0} \int_{\Omega}\left(1-\chi_{h}\right)\left|\frac{\left[\left(\nabla_{h} y^{(h)}\right)^{\mathrm{T}} \nabla_{h} y^{(h)}\right]^{1 / 2}-\mathrm{Id}}{h^{2}}\right|^{2} \mathrm{~d} x \leqslant 0 .
$$

This concludes the proof of the strong convergence of the scaled nonlinear strain.

To establish the strong convergence of $\left(\phi_{1}^{(h)}\right)$ in $W^{1,2}(\Omega)$, we first prove that

$$
\frac{1}{h^{2}} \operatorname{dist}\left(\nabla_{h} \tilde{y}^{(h)}, \operatorname{SO}(3)\right) \rightarrow|\widetilde{G}| \quad \text { in } L^{2}(\Omega) \text {. }
$$

From the definition of $G^{(h)}$ it follows that

$$
\begin{aligned}
\frac{1}{h^{2}} \chi_{h} \operatorname{dist}\left(\nabla_{h} \tilde{y}^{(h)}, \operatorname{SO}(3)\right) & =\frac{1}{h^{2}} \chi_{h} \operatorname{dist}\left(\operatorname{Id}+h^{2} G^{(h)}, \operatorname{SO}(3)\right) \\
& =\chi_{h}\left|\operatorname{sym} G^{(h)}\right|+\chi_{h} \mathrm{O}\left(\frac{1}{h^{2}}\left|G^{(h)}\right|^{2}\right) .
\end{aligned}
$$

By (5.9) and the fact that $\chi_{h}\left(1 / h^{2}\right)\left|G^{(h)}\right|^{2}$ is bounded by $h$, we deduce that

$$
\frac{1}{h^{2}} \chi_{h} \operatorname{dist}\left(\nabla_{h} \tilde{y}^{(h)}, \operatorname{SO}(3)\right) \rightarrow|\widetilde{G}| \quad \text { in } L^{2}(\Omega)
$$

In combination with (5.10) this yields (5.11). In particular, the convergence (5.11) implies that the sequence $\left(\left(1 / h^{4}\right) \operatorname{dist}^{2}\left(\nabla_{h} \tilde{y}^{(h)}, \mathrm{SO}(3)\right)\right)$ is equi-integrable. By a refined version of Theorem 2.1 (see Proposition 5.2 below) this implies that

$$
\left|G^{(h)}\right|^{2}=\frac{1}{h^{4}}\left|\left(R^{(h)}\right)^{\mathrm{T}} \nabla_{h} \tilde{y}^{(h)}-\mathrm{Id}\right|^{2} \quad \text { is equi-integrable. }
$$

By (5.9) and (5.12) we have that

$$
\operatorname{sym} G^{(h)} \rightarrow \widetilde{G} \quad \text { strongly in } L^{2}(\Omega) .
$$

Since $R^{(h)} \rightarrow$ Id strongly in $L^{\infty}$, we obtain

$$
\left(R^{(h)}-\mathrm{Id}\right) G^{(h)} \rightarrow 0 \quad \text { strongly in } L^{2}(\Omega) .
$$

Thus,

$$
\frac{1}{h^{2}} \operatorname{sym}\left(\nabla_{h} \tilde{y}^{(h)}-R^{(h)}\right)=\operatorname{sym}\left(R^{(h)} G^{(h)}\right) \rightarrow \widetilde{G} \quad \text { strongly in } L^{2}(\Omega) .
$$

Now, since we can decompose $\phi_{1,1}^{(h)}$ as

$$
\phi_{1,1}^{(h)}=\frac{\tilde{y}_{1,1}^{(h)}-R_{11}^{(h)}}{h^{2}}+\frac{R_{11}^{(h)}-1}{h^{2}},
$$

we have by (5.14) and property (e) of Theorem 2.2 that $\left(\phi_{1,1}^{(h)}\right)$ is strongly convergent in $L^{2}(\Omega)$. The strong convergence of $\left(\phi_{1, k}^{(h)}\right)$ for $k=2,3$ follows from property (d) of Theorem 2.2. This concludes the proof.

Proposition 5.2. Let $\left(y^{(h)}\right)$ be a sequence in $W^{1,2}\left(\Omega ; \mathbb{R}^{3}\right)$ such that

$$
\operatorname{dist}\left(\nabla_{h} y^{(h)}, \mathrm{SO}(3)\right) \leqslant h^{2}(M+f)
$$


where $M \in \mathbb{R}, M \geqslant 0$, and $f \in L^{2}(\Omega)$. Let $R^{(h)}$ be the map constructed in Theorem 2.2. Then

$$
\left|\nabla_{h} y^{(h)}-R^{(h)}\right| \leqslant h^{2}\left(G_{1}+G_{2}\right)
$$

with

$$
\left\|G_{1}\right\|_{L^{p}(\Omega)} \leqslant C M \quad \text { for some } p>2, \quad\left\|G_{2}\right\|_{L^{2}(\Omega)} \leqslant C\|f\|_{L^{2}(\Omega)} .
$$

In particular, if $h^{-4} \operatorname{dist}\left(\nabla_{h} y^{(h)}, \mathrm{SO}(3)\right)$ is equi-integrable, then $h^{-4}\left|\nabla_{h} y^{(h)}-R^{(h)}\right|^{2}$ is equi-integrable.

For a proof we refer to [8].

\subsection{Comparison with linear elasticity}

The result of Theorem 5.1 can be read as follows. The sequence $\left(\phi^{(h)}\right)$, which describes the scaled deviation of $\tilde{y}^{(h)}$ from the identity, behaves asymptotically as the sequence $\left(\hat{\phi}^{(h)}\right)$ defined by

$$
\begin{aligned}
& \hat{\phi}_{1}^{(h)}=u-x_{2} v_{2,1}-x_{3} v_{3,1}+h \beta_{1}, \\
& \hat{\phi}_{k}^{(h)}=v_{k}+h w x_{k}^{\perp}+h^{2} \beta_{k} \quad(k=2,3),
\end{aligned}
$$

in the following sense. We set for $\phi \in W^{1,2}\left(\Omega ; \mathbb{R}^{3}\right)$

$$
D^{(h)}(\phi):=\left(\begin{array}{cc}
\phi_{1,1} & \frac{1}{h} \phi_{1, k} \\
\frac{1}{h} \phi_{j, 1} & \frac{1}{h^{2}} \phi_{j, k}
\end{array}\right), \quad j, k \in\{2,3\} .
$$

Then it is easy to see that the scaled nonlinear strain can be expressed in terms of $\phi^{(h)}$ as

$$
\frac{\left[\left(\nabla_{h} \tilde{y}^{(h)}\right)^{\mathrm{T}} \nabla_{h} \tilde{y}^{(h)}\right]^{1 / 2}-\mathrm{Id}}{h^{2}}=\operatorname{sym} D^{(h)}\left(\phi^{(h)}\right)+\frac{1}{2} h^{2}\left[D^{(h)}\left(\phi^{(h)}\right)\right]^{\mathrm{T}} D^{(h)}\left(\phi^{(h)}\right)+\mathrm{O}\left(h^{2}\left|\operatorname{sym} D^{(h)}\left(\phi^{(h)}\right)\right|^{2}\right),
$$

and that

$$
\operatorname{sym} D^{(h)}\left(\hat{\phi}^{(h)}\right)+\frac{1}{2} h^{2}\left[D^{(h)}\left(\hat{\phi}^{(h)}\right)\right]^{\mathrm{T}} D^{(h)}\left(\hat{\phi}^{(h)}\right)=\widetilde{G}+\mathrm{O}(h)
$$

provided $\beta \in W^{1,2}\left(\Omega ; \mathbb{R}^{3}\right)$. In this case, the convergence result of Theorem 5.1 and the fact that $h \operatorname{sym} D^{(h)}\left(\phi^{(h)}\right) \rightarrow$ $\operatorname{sym} A=0$ in $L^{2}(\Omega)$ imply that

$$
\begin{aligned}
& \left(\operatorname{sym} D^{(h)}\left(\phi^{(h)}\right)+\frac{1}{2} h^{2}\left[D^{(h)}\left(\phi^{(h)}\right)\right]^{\mathrm{T}} D^{(h)}\left(\phi^{(h)}\right)\right) \\
& \quad-\left(\operatorname{sym} D^{(h)}\left(\hat{\phi}^{(h)}\right)+\frac{1}{2} h^{2}\left[D^{(h)}\left(\hat{\phi}^{(h)}\right)\right]^{\mathrm{T}} D^{(h)}\left(\hat{\phi}^{(h)}\right)\right) \rightarrow 0 \quad \text { strongly in } L^{1}(\Omega) .
\end{aligned}
$$

The condition $\beta \in W^{1,2}\left(\Omega ; \mathbb{R}^{3}\right)$ corresponds to higher regularity of the solution, as shown in Lemmas 5.3 and 5.4 below.

The comparison of $\phi^{(h)}$ and $\hat{\phi}^{(h)}$ generalizes to the nonlinear setting an earlier result by Murat and Sili in the context of linearized elasticity. More precisely, Murat and Sili have studied in [13] the asymptotic behaviour of the solution $\varphi^{(h)}$ of a linearized elasticity problem in an inhomogeneous cylinder, whose diameter $h$ tends to 0 . They show that $\varphi^{(h)}$ has the same asymptotic behaviour of

$$
\hat{\varphi}^{(h)}:=\varphi+h \psi+h^{2} \zeta
$$

in the following sense:

$$
\begin{aligned}
& \varphi^{(h)} \rightarrow \varphi \quad \text { strongly in } W^{1,2}, \\
& e^{(h)}\left(\varphi^{(h)}\right)-e^{(h)}\left(\hat{\varphi}^{(h)}\right) \rightarrow 0 \quad \text { strongly in } L^{2},
\end{aligned}
$$


where $e^{(h)}(f):=\operatorname{sym} D^{(h)}(f)$. The functions $\varphi, \psi, \zeta$ enjoy the following properties: $\varphi$ satisfies the BernoulliNavier equation, i.e., there exist $u \in W^{1,2}(0, L)$ and $v_{k} \in W^{2,2}(0, L)$ such that

$$
\varphi_{1}=u-x_{2} v_{2,1}-x_{3} v_{3,1}, \quad \varphi_{k}=v_{k}
$$

the function $\psi_{1}$ belongs to $L^{2}\left(\Omega ; \mathbb{R}^{3}\right)$ with derivatives $\psi_{1,2}, \psi_{1,3}$ in $L^{2}\left(\Omega ; \mathbb{R}^{3}\right)$, while there exists a function $w \in W^{1,2}(0, L)$ such that

$$
\psi_{2}(x)=-w\left(x_{1}\right) x_{3}, \quad \psi_{3}(x)=w\left(x_{1}\right) x_{2} ;
$$

finally, the function $\zeta$ belongs to the space

$$
\left\{\theta \in L^{2}\left(\Omega ; \mathbb{R}^{3}\right): \theta_{1}=0, \theta_{k, j} \in L^{2}(\Omega) \text { for } j, k=2,3, \int_{S}\left(x_{3} \theta_{2}-x_{2} \theta_{3}\right) \mathrm{d} x_{2} \mathrm{~d} x_{3}=0\right\} .
$$

This linear asymptotic result is in agreement with Theorem 5.1. Indeed, $\varphi^{(h)}$, as solution of a linearized elasticity problem, is defined as a suitable rescaling of the deviation of the deformation $y^{(h)}$ from the identity (which corresponds to $\phi^{(h)}$ in our notation), while $e^{(h)}\left(\varphi^{(h)}\right)$ is the linearized strain. Moreover, the asymptotic development $\hat{\varphi}^{(h)}$ found by Murat and Sili has exactly the same structure of the asymptotic development $\hat{\phi}^{(h)}$ found in the nonlinear case.

We conclude the section with two lemmas showing that higher regularity of $\beta$ is related to higher regularity of solutions.

Lemma 5.3. Assume that the function $x_{1} \mapsto \frac{\partial^{2} W}{\partial F^{2}}\left(\left(x_{1}, x^{\prime}\right)\right.$, Id $)$ is differentiable for a.e. $x^{\prime} \in S$ and

$$
\left|\frac{\partial}{\partial x_{1}}\left(\frac{\partial^{2} W}{\partial F^{2}}\right)(x, \mathrm{Id})\right| \leqslant \widetilde{C} \quad \text { for a.e. } x \in \Omega \text {. }
$$

Let $u \in W^{2,2}(0, L)$ and $A \in W^{2,2}\left((0, L) ; \mathbb{M}^{3 \times 3}\right)$. Let $\alpha^{\min }\left(x_{1}, \cdot\right)$ denote the solution in $V$ of the problem (4.1) defining $Q\left(x_{1}, u_{, 1}+\frac{1}{2}\left(v_{2,1}^{2}+v_{3,1}^{2}\right), A_{, 1}\right)$. Then $\alpha^{\text {min }}$ belongs to $W^{1,2}\left(\Omega ; \mathbb{R}^{3}\right)$, as well as the function $\beta$ determined by (5.8) and (5.7).

Proof. Let $I^{\prime}$ be an open interval compactly contained in $(0, L)$. Let $x_{1} \in I^{\prime}$ and $\varepsilon>0$. For any function $f: \Omega \rightarrow \mathbb{R}^{N}$ we set

$$
\Delta_{\varepsilon} f(x):=\frac{1}{\varepsilon}\left(f\left(x_{1}+\varepsilon, x^{\prime}\right)-f(x)\right) .
$$

Using the Euler-Lagrange equation (4.3) we obtain

$$
\begin{aligned}
\int_{S} & \sum_{h, k=2,3}\left(B^{h k}\left(x_{1}+\varepsilon, x^{\prime}\right) \Delta_{\varepsilon} \alpha_{, k}^{\min }(x), \varphi_{, h}\left(x^{\prime}\right)\right) \mathrm{d} x^{\prime} \\
= & -\int_{S} \sum_{h=2,3}\left(\Delta_{\varepsilon} B^{h 1}(x) g(x)+B^{h 1}\left(x_{1}+\varepsilon, x^{\prime}\right) \Delta_{\varepsilon} g(x), \varphi_{, h}\left(x^{\prime}\right)\right) \mathrm{d} x^{\prime} \\
& -\int_{S} \sum_{h, k=2,3}\left(\Delta_{\varepsilon} B^{h k}(x) \alpha_{, k}^{\min }(x), \varphi_{, h}\left(x^{\prime}\right)\right) \mathrm{d} x^{\prime}
\end{aligned}
$$

where $\varphi$ is any test function in $W^{1,2}\left(S ; \mathbb{R}^{3}\right)$ and

$$
g(x):=A_{, 1}\left(x_{1}\right)\left(\begin{array}{c}
0 \\
x_{2} \\
x_{3}
\end{array}\right)+\left(u_{, 1}\left(x_{1}\right)+\frac{1}{2} v_{2,1}^{2}\left(x_{1}\right)+\frac{1}{2} v_{3,1}^{2}\left(x_{1}\right)\right) e_{1} .
$$


Taking $\Delta_{\varepsilon} \alpha^{\min }\left(x_{1}, \cdot\right)$ as test function in (5.16) and arguing as in the proof of (4.5), we obtain that there exists a constant $C$ (independent of $x_{1}, I^{\prime}$, and $\varepsilon$ ) such that

$$
\begin{aligned}
\left\|\Delta_{\varepsilon} \alpha_{, 2}^{\min }\right\|_{L^{2}(S)}^{2}+\left\|\Delta_{\varepsilon} \alpha_{, 3}^{\min }\right\|_{L^{2}(S)}^{2} & \leqslant C \sum_{h=2,3}\left\|\Delta_{\varepsilon} B^{h 1} g+B^{h 1} \Delta_{\varepsilon} g+\sum_{k=2,3} \Delta_{\varepsilon} B^{h k} \alpha_{, k}^{\min }\right\|_{L^{2}(S)}^{2} \\
& \leqslant C\left(\|g\|_{L^{2}(S)}^{2}+\left\|\Delta_{\varepsilon} g\right\|_{L^{2}(S)}^{2}+\left\|\alpha_{, 2}^{\min }\right\|_{L^{2}(S)}^{2}+\left\|\alpha_{, 3}^{\min }\right\|_{L^{2}(S)}^{2}\right) .
\end{aligned}
$$

By the Sobolev-Poincaré inequality we know that

$$
\left\|\Delta_{\varepsilon} \alpha^{\min }\left(x_{1}, \cdot\right)\right\|_{L^{2}(S)} \leqslant C\left(\left\|\Delta_{\varepsilon} \alpha_{, 2}^{\min }\right\|_{L^{2}(S)}^{2}+\left\|\Delta_{\varepsilon} \alpha_{, 3}^{\min }\right\|_{L^{2}(S)}^{2}\right) .
$$

Integrating both sides on $I^{\prime}$ and using (5.18), we have

$$
\left\|\Delta_{\varepsilon} \alpha^{\min }\right\|_{L^{2}\left(I^{\prime} \times S\right)} \leqslant C\left(\|g\|_{L^{2}(\Omega)}^{2}+\left\|\Delta_{\varepsilon} g\right\|_{L^{2}\left(I^{\prime} \times S\right)}^{2}+\left\|\alpha_{, 2}^{\min }\right\|_{L^{2}(\Omega)}^{2}+\left\|\alpha_{, 3}^{\min }\right\|_{L^{2}(\Omega)}^{2}\right)
$$

To conclude it is enough to show that the right-hand side is bounded by a constant independent of $I^{\prime}$ and $\varepsilon$. The only term for which this is not trivial is the quantity $\left\|\Delta_{\varepsilon} g\right\|_{L^{2}\left(I^{\prime} \times S\right)}$. By (5.17) we have

$$
g_{, 1}=A_{, 11}\left(\begin{array}{c}
0 \\
x_{2} \\
x_{3}
\end{array}\right)+\left(u, 11+v_{2,1} v_{2,11}+v_{3,1} v_{3,11}\right) e_{1} .
$$

From the regularity assumptions on $A$ and $u$ it follows that $g, 1$ belongs to $L^{2}\left(\Omega ; \mathbb{R}^{3}\right)$. This implies the required bound on $\left\|\Delta_{\varepsilon} g\right\|_{L^{2}\left(I^{\prime} \times S\right)}$.

Let $f_{2}, f_{3} \in L^{2}(0, L)$. We introduce the functional

$$
J^{0}\left(u, v_{2}, v_{3}, w\right):=I^{0}\left(u, v_{2}, v_{3}, w\right)-\int_{0}^{L} \sum_{k=2,3} f_{k} v_{k} \mathrm{~d} x_{1}
$$

for every $u \in W^{1,2}(0, L), v_{2}, v_{3} \in W^{2,2}(0, L)$, and $w \in W^{1,2}(0, L)$.

Lemma 5.4. Assume that the function $x_{1} \mapsto \frac{\partial^{2} W}{\partial F^{2}}\left(\left(x_{1}, x^{\prime}\right)\right.$, Id $)$ is differentiable for a.e. $x^{\prime} \in S$ and

$$
\left|\frac{\partial}{\partial x_{1}}\left(\frac{\partial^{2} W}{\partial F^{2}}\right)(x, \mathrm{Id})\right| \leqslant \widetilde{C} \quad \text { for a.e. } x \in \Omega .
$$

Let $f_{2}, f_{3} \in L^{2}(0, L)$ and let $\left(u, v_{2}, v_{3}, w\right)$ be a minimizer for $J^{0}$. Then $u, w \in W^{2, \infty}(0, L)$ and $v_{2}, v_{3} \in$ $W^{3, \infty}(0, L)$. In particular, $A \in W^{2, \infty}\left((0, L) ; \mathbb{M}^{3 \times 3}\right)$.

Proof. Let $\widehat{B}:(0, L) \rightarrow \mathbb{M}^{3 \times 3}$ be the symmetric matrix associated to the quadratic form $Q\left(x_{1}, \cdot\right)$ defined in (4.1); thus, we can express $Q$ as

$$
Q\left(x_{1}, t, F\right)=\left(\widehat{B}\left(x_{1}\right)\left(\begin{array}{c}
t \\
F_{12} \\
F_{13} \\
F_{32}
\end{array}\right),\left(\begin{array}{c}
t \\
F_{12} \\
F_{13} \\
F_{32}
\end{array}\right)\right) \forall t \in \mathbb{R}, \forall F \in \mathbb{M}_{\text {skew }}^{3 \times 3} .
$$

The matrix $\widehat{B}$ is uniformly positive definite by (4.4). Moreover, from the assumption of differentiability of $\partial^{2} W / \partial F^{2}$ and (5.20), it follows that $\widehat{B}$ is differentiable with respect to $x_{1}$ and its derivative is bounded on $(0, L)$. We will denote the $j$-th row of $\widehat{B}$ by $\widehat{B}_{j}$. 
For simplicity of notation we introduce the function $\zeta \in L^{2}\left((0, L) ; \mathbb{R}^{4}\right)$ defined by

$$
\zeta\left(x_{1}\right):=\left(\begin{array}{c}
u_{, 1}+\frac{1}{2} v_{2,1}^{2}+\frac{1}{2} v_{3,1}^{2} \\
v_{2,11} \\
v_{3,11} \\
w_{, 1}
\end{array}\right)
$$

If $\left(u, v_{2}, v_{3}, w\right)$ is a minimizer of $J^{0}$, then the following Euler-Lagrange equations hold:

$$
\begin{aligned}
& \int_{0}^{L} \varphi_{1,1} \widehat{B}_{1} \zeta \mathrm{d} x_{1}=0, \\
& \int_{0}^{L} \varphi_{2,11} \widehat{B}_{2} \zeta \mathrm{d} x_{1}=-\int_{0}^{L} \varphi_{2,1} v_{2,1} \widehat{B}_{1} \zeta \mathrm{d} x_{1}+\int_{0}^{L} f_{2} \varphi_{2} \mathrm{~d} x_{1}, \\
& \int_{0}^{L} \varphi_{3,11} \widehat{B}_{3} \zeta \mathrm{d} x_{1}=-\int_{0}^{L} \varphi_{3,1} v_{3,1} \widehat{B}_{1} \zeta \mathrm{d} x_{1}+\int_{0}^{L} f_{3} \varphi_{3} \mathrm{~d} x_{1}, \\
& \int_{0}^{L} \varphi_{4,1} \widehat{B}_{4} \zeta \mathrm{d} x_{1}=0
\end{aligned}
$$

for every $\varphi_{i} \in C^{\infty}(0, L)$. From these equations we want to deduce some higher regularity of $\zeta$.

From (5.21) it follows that there exists a constant $c_{1} \in \mathbb{R}$ such that

$$
\widehat{B}_{1}\left(x_{1}\right) \zeta\left(x_{1}\right)=c_{1} \text { for a.e. } x_{1} \in(0, L) \text {. }
$$

Analogously, (5.24) implies that there exists a constant $c_{4} \in \mathbb{R}$ such that

$$
\widehat{B}_{4}\left(x_{1}\right) \zeta\left(x_{1}\right)=c_{4} \quad \text { for a.e. } x_{1} \in(0, L) \text {. }
$$

Using (5.25) in (5.22) and (5.23), we have for $k=2,3$

$$
\int_{0}^{L} \varphi_{k, 11} \widehat{B}_{k} \zeta \mathrm{d} x_{1}=\int_{0}^{L}\left(f_{k} \varphi_{k}-c_{1} v_{k, 1} \varphi_{k, 1}\right) \mathrm{d} x_{1}=\int_{0}^{L}\left(f_{k}+c_{1} v_{k, 11}\right) \varphi_{k} \mathrm{~d} x_{1} \quad \forall \varphi_{k} \in C_{0}^{\infty}(0, L) .
$$

This implies that the second derivative of $\widehat{B}_{k} \zeta$ belongs to $L^{2}(0, L)$ and coincides with $f_{k}+c_{1} v_{k, 11}$ almost everywhere. Therefore, there exists $g_{k} \in W^{2,2}(0, L)$ such that $g_{k, 11}=f_{k}$ and

$$
\widehat{B}_{k}\left(x_{1}\right) \zeta\left(x_{1}\right)=g_{k}\left(x_{1}\right) \text { for a.e. } x_{1} \in(0, L) \text {. }
$$

Combining together (5.25), (5.26), and (5.27), we have

$$
\widehat{B}\left(x_{1}\right) \zeta\left(x_{1}\right)=g\left(x_{1}\right) \in W^{2,2}\left((0, L) ; \mathbb{R}^{4}\right),
$$

where we have set $g:=c_{1} e_{1}+g_{2} e_{2}+g_{3} e_{3}+c_{4} e_{4}$. Since $\widehat{B}$ belongs to $W^{1, \infty}$ and is uniformly coercive, the inverse $\widehat{B}^{-1}$ is still in $W^{1, \infty}$; therefore,

$$
\zeta\left(x_{1}\right)=\left(\widehat{B}^{-1}\right)\left(x_{1}\right) g\left(x_{1}\right) \in W^{1, \infty}\left((0, L) ; \mathbb{R}^{4}\right) .
$$

This immediately implies that $v_{k} \in W^{3, \infty}(0, L)$ and $w \in W^{2, \infty}(0, L)$. Moreover, we have that $u_{, 1}+\frac{1}{2} v_{2,1}^{2}+\frac{1}{2} v_{3,1}^{2}$ belongs to $W^{1, \infty}(0, L)$. Since $v_{k, 1}^{2} \in W^{1, \infty}(0, L)$, we can conclude that $u_{, 1}$ belongs to $W^{1, \infty}(0, L)$ too. This finishes the proof of the lemma. 
Remark 5.5. The functional $J^{0}$ defined in (5.19) can be obtained as $\Gamma$-limit of the energies $\left(1 / h^{4}\right) I^{(h)}$ by adding a term describing transversal body forces of order $h^{3}$ and by imposing a boundary condition which eliminates rigid motions of the body.

\section{Acknowledgements}

Both authors were supported by the TMR Network "Phase transitions in crystalline solids" (FMRX-CT980229). We thank François Murat for explaining us the work in [13], for his suggestion to look for a nonlinear counterpart, and for many inspiring discussions.

\section{References}

[1] E. Acerbi, G. Buttazzo, D. Percivale, A variational definition for the strain energy of an elastic string, J. Elasticity 25 (1991) $137-148$.

[2] S.S. Antman, The Theory of Rods, in: Handbuch der Physik, vol. VIa, Springer-Verlag, 1972.

[3] S.S. Antman, Nonlinear Problems of Elasticity, Springer-Verlag, New York, 1995.

[4] A. Cimetière, G. Geymonat, H. Le Dret, A. Raoult, Z. Tutek, Asymptotic theory and analysis for displacements and stress distribution in nonlinear elastic straight slender rods, J. Elasticity 19 (1988) 111-161.

[5] G. Dal Maso, An Introduction to $\Gamma$-convergence, Birkhäuser, Boston, 1993.

[6] G. Friesecke, R.D. James, S. Müller, A theorem on geometric rigidity and the derivation of nonlinear plate theory from three-dimensional elasticity, Comm. Pure Appl. Math. 55 (2002) 1461-1506.

[7] G. Friesecke, R.D. James, S. Müller, The Föppl von Kármán plate theory as a low energy $\Gamma$-limit of nonlinear elasticity, C. R. Acad. Sci. Paris, Ser. I 335 (2002) 201-206.

[8] G. Friesecke, R.D. James, S. Müller, A hierarchy of plate models derived from nonlinear elasticity by $\Gamma$-convergence, in preparation.

[9] G. Kirchhoff, Über das Gleichgewicht und die Bewegungen eines unendlich dünnen Stabes, J. Reine Angew. Math. (Crelle) 56 (1859) 285-313.

[10] A. Mielke, On Saint-Venant's problem for an elastic strip, Proc. Roy. Soc. Edinburgh Sect. A 110 (1988) 161-181.

[11] A. Mielke, Saint-Venant's problem and semi-inverse solutions in nonlinear elasticity, Arch. Rational Mech. Anal. 102 (1988) $205-229$.

[12] M.G. Mora, S. Müller, Derivation of the nonlinear bending-torsion theory for inextensible rods by $\Gamma$-convergence, Calc. Var., in press.

[13] F. Murat, A. Sili, Comportement asymptotique des solutions du système de l'élasticité linéarisée anisotrope hétérogène dans des cylindres minces, C. R. Acad. Sci. Paris, Sér. I Math. 328 (1999) 179-184.

[14] F. Murat, A. Sili, Effets non locaux dans le passage 3d-1d en élasticité linéarisée anisotrope hétérogène, C. R. Acad. Sci. Paris, Sér. I Math. 330 (2000) 745-750.

[15] O.A. Oleinik, A.S. Shamaev, G.A. Yosifian, Mathematical Problems in Elasticity and Homogenization, North-Holland, 1992.

[16] O. Pantz, Le modèle de poutre inextensionnelle comme limite de l'élasticité non-linéaire tridimensionnelle, Preprint, 2002.

[17] P. Villaggio, Mathematical Models for Elastic Structures, Cambridge University Press, 1997. 